\title{
Ballistic behavior for random Schrödinger operators on the Bethe strip
}

\author{
Abel Klein ${ }^{1}$ and Christian Sadel
}

\begin{abstract}
The Bethe strip of width $m$ is the Cartesian product $\mathbb{B} \times\{1, \ldots, m\}$, where $\mathbb{B}$ is the Bethe lattice (Cayley tree). We consider Anderson-like Hamiltonians $H_{\lambda}=\frac{1}{2} \Delta \otimes 1+$ $1 \otimes A+\lambda \mathcal{V}$ on a Bethe strip with connectivity $K \geq 2$, where $A$ is an $m \times m$ symmetric matrix, $\mathcal{V}$ is a random matrix potential, and $\lambda$ is the disorder parameter. Under certain conditions on $A$ and $K$, for which we previously proved the existence of absolutely continuous spectrum for small $\lambda$, we now obtain ballistic behavior for the spreading of wave packets evolving under $H_{\lambda}$ for small $\lambda$.
\end{abstract}

Mathematics Subject Classification (2010). Primary 82B44; Secondary 47B80, 60H25.

Keywords. Random Schrödinger operators, Anderson model, spreading of wave packets, ballistic behavior, Bethe strip.

\section{Contents}

1 Introduction . . . . . . . . . . . . . . . . . . . . 409

2 Supersymmetric methods . . . . . . . . . . . . . . . . . 414

3 More supersymmetric identities . . . . . . . . . . . . . . . . . . 422

4 Averages of the matrix Green's function . . . . . . . . . . . . . . 431

5 The proof of Theorem $1.2 \ldots \ldots . \ldots . \ldots 437$

References ...................... . . 440

\section{Introduction}

The Bethe lattice (or Cayley tree) $\mathbb{B}$ is an infinite connected graph with no closed loops where each vertex has $K+1$ neighbors. $K \in \mathbb{N}$ is called the connectivity of $\mathbb{B}$. The Bethe strip of width $m$ is the Cartesian product $\mathbb{B} \times \mathcal{I}$, where $\mathcal{I}=\{1, \ldots, m\}$. The distance between two sites $x$ and $y$ of $\mathbb{B}$, denoted by $d(x, y)$, is equal to the length of the shortest path connecting $x$ and $y$ in $\mathbb{B}$. The $\ell^{2}$ space of functions on the

\footnotetext{
${ }^{1}$ Abel Klein was supported in part by the NSF under grant DMS-1001509.
} 
Bethe strip, $\ell^{2}(\mathbb{B} \times \mathcal{I})$, can be identified with the tensor product $\ell^{2}(\mathbb{B}) \otimes \mathbb{C}^{m}$, with the direct sum $\bigoplus_{x \in \mathbb{B}} \mathbb{C}^{m}$, and with $\ell^{2}\left(\mathbb{B}, \mathbb{C}^{m}\right)=\left\{u: \mathbb{B} \mapsto \mathbb{C}^{m}: \sum_{x \in \mathbb{B}}\|u(x)\|^{2}<\infty\right\}$, the space of $\mathbb{C}^{m}$-valued $\ell^{2}$ functions on $\mathbb{B}$, i.e.

$$
\ell^{2}(\mathbb{B} \times \mathcal{I}) \cong \ell^{2}(\mathbb{B}) \otimes \mathbb{C}^{m} \cong \bigoplus_{x \in \mathbb{B}} \mathbb{C}^{m} \cong \ell^{2}\left(\mathbb{B}, \mathbb{C}^{m}\right) .
$$

As in [28] we consider the family of random Hamiltonians on $\ell^{2}(\mathbb{B} \times \mathfrak{I})$ given by

$$
H_{\lambda}=\frac{1}{2} \Delta \otimes 1+1 \otimes A+\lambda \mathcal{V} .
$$

Here $\Delta$ denotes the centered Laplacian on $\ell^{2}(\mathbb{B})$, which has spectrum $\sigma(\Delta)=$ $[-2 \sqrt{K}, 2 \sqrt{K}]$ (see, e.g., [1]). We use $\frac{1}{2} \Delta$ in the definition of $H_{\lambda}$ to simplify some formulas. $A \in \operatorname{Sym}(m)$ denotes the "free vertical operator" on the Bethe strip, where $\operatorname{Sym}(m) \cong \mathbb{R}^{\frac{1}{2} m(m+1)}$ is the set of real symmetric $m \times m$ matrices. $\mathcal{V}$ is the random matrix-potential given by $\mathcal{V}=\bigoplus_{x \in \mathbb{B}} V(x)$ on $\bigoplus_{x \in \mathbb{B}} \mathbb{C}^{m}$, where $\{V(x)\}_{x \in \mathbb{B}}$ are independent identically distributed $\operatorname{Sym}(m)$-valued random variables with common probability distribution $\mu$. The coefficient $\lambda$ is a real parameter called the disorder. In particular, for $u \in \ell^{2}\left(\mathbb{B}, \mathbb{C}^{m}\right)$ we have

$$
\left(H_{\lambda} u\right)(x)=\frac{1}{2} \sum_{\substack{y \in \mathbb{B} \\ d(x, y)=1}} u(y)+A u(x)+\lambda V(x) u(x) \quad \text { for all } x \in \mathbb{B} .
$$

An important special case of this model is the Anderson model on the product graph $\mathbb{B} \times \mathbb{G}$, where $\mathbb{G}$ is a finite graph with $m$ labeled vertices. If $A_{\mathbb{G}}$ is the adjacency matrix of the graph $\mathbb{G}$, i.e. $\left(A_{\mathbb{G}}\right)_{k, \ell}$ denotes the number of edges between $k \in \mathbb{G}$ and $\ell \in \mathbb{G}$, then $\Delta \otimes 1+1 \otimes A_{\mathbb{G}}$ is the adjacency operator on the product graph $\mathbb{B} \times \mathbb{G}$. If in (1.1) we take $A=\frac{1}{2} A_{\mathbb{G}}$ and $\mu$ supported by the diagonal matrices, with the diagonal entries being independent identically distributed, then $H_{\lambda}$ is the Anderson model on the product graph $\mathbb{B} \times \mathbb{G}$. Another special case is the Wegner $m$-orbital model on the Bethe lattice, obtained by setting $A=0$ and letting $\mu$ be the probability distribution of the Gaussian Orthogonal Ensemble (GOE). This model was introduced by Wegner [36] on the lattice $\mathbb{Z}^{d}$, where he studied the limit $m \rightarrow \infty$.

There is a widely accepted picture for the Anderson model on the lattice $\mathbb{Z}^{d}$, for $d=1$ and $d=2$ and any $\lambda \neq 0$, and for $d \geq 3$ and large $\lambda$, there is only exponential localization, i.e. pure point spectrum with exponentially decaying eigenfunctions. For $d \geq 3$ and small $\lambda \neq 0$, in addition to exponential localization at the spectral edges, the existence of extended states, i.e. absolutely continuous spectrum, is expected but not yet proven. By now, localization in dimension $d=1$, [17], [31], and [8], in quasi-one dimensional models (the strip), [32] and [27], and in any dimension at the spectral edges or at high disorder (i.e. large $\lambda$ ), [16], [15], [9], [34], [8], [10], [21], [3], [2], [35], and [30], is very well understood. Localization in dimension $d=2$ at low disorder as well as absolutely continuous spectrum in dimensions $d \geq 3$ at small disorder remain open problems. 
Localization and delocalization can also be observed by examining the quantum mechanical dynamical behavior, as seen in the spreading of wave packets under the time evolution. Localization corresponds to effective non-spreading of wave packets (dynamical localization). If $d \geq 3$, diffusive behavior for the spreading of wave packets is expected for small $\lambda$. This is analogous to the random walk in dimension $d \geq 3$, which is diffusive.

So far, the existence of absolutely continuous spectrum has only been proven for the Anderson model on the Bethe lattice, the Bethe strip and similar tree like structures. The first rigorous proof of absolutely continuous spectrum for the Anderson model in the Bethe lattice was obtained by Klein, [22], [25], and [23], using a supersymmetric transfer matrix method. These methods were extended to the Bethe strip in our previous work [28], where we proved the existence of absolutely continuous spectrum in the Bethe strip.

In addition, Klein showed that the supersymmetric method also yielded ballistic behavior in the Bethe lattice [24]. (Note that the random walk on the Bethe lattice is ballistic.) In this paper we extend these methods to the Bethe strip, proving ballistic behavior for the Anderson model in the Bethe strip.

Different techniques to obtain absolutely continuous spectrum for the Anderson model on the Bethe lattice and similar tree like structures have been developed in [4], [13], [12], [18], [19], [14], and [5]. The hyperbolic geometry methods of [13] and [18] were extended to the Anderson model on a Bethe strip of connectivity $K=2$ and width $m=2$ in [12]. However, up to now the results of [24] remained the only proof of dynamical delocalization for the Anderson model.

Our proof of absolutely continuous spectrum for the Anderson model on the Bethe strip [28] used the approach of [25] combined with the supersymmetric formalism for the strip developed in [29]. But although the present paper uses the approach of [24], which relied on the methods and results of [25], it does not suffice for us to rely on the methods of [28]. The approach is based on a supersymmetric transfer matrix formalism for the Green's function. In [24] this lead to the study of certain operators on an $L^{2}$-space. On the Bethe strip this is much more complicated, and requires an augmentation of the supersymmetric formalism, with the derivation of new supersymmetric identities and the introduction of new Hilbert and Banach spaces of supersymmetric functions. This is done in Section 3; the key results being Theorem 3.3 and Corollary 3.4. For the Bethe lattice, i.e. $m=1$, the Grassmann variables can be integrated out explicitly, and the Hilbert space $\mathbb{H}$ (see (3.18)) reduces

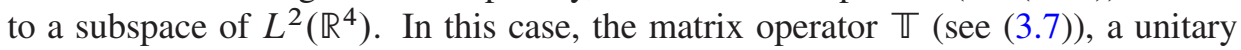
operator on $\mathbb{H}$ made out of differential operators and the Fourier transform, reduces to the Fourier transform. (The differential operators do not appear when $m=1$; this can been seen from the definition (3.7) and the relation (2.26), where there are no derivatives on the right hand side when $m=n=1$.)

In this article, as in [28], we always make the following assumptions.

\section{Assumptions}

(I) $K \geq 2$, so $\mathbb{B}$ is not the line $\mathbb{R}$. 
(II) The common probability distribution $\mu$ of the $\operatorname{Sym}(m)$-valued random variables $\{V(x)\}_{x \in \mathbb{B}}$ has finite (mixed) moments of all orders. In particular, the characteristic function of $\mu$,

$$
h(M):=\int_{\operatorname{Sym}(m)} \mathrm{e}^{-i \operatorname{Tr}(M V)} d \mu(V) \text { for } M \in \operatorname{Sym}(m),
$$

is a $C^{\infty}$ function on $\operatorname{Sym}(m)$ with bounded derivatives.

(III) Let $a_{\min }:=a_{1} \leq a_{2} \leq \ldots \leq a_{m}=: a_{\max }$ be the eigenvalues of the "free vertical operator" $A$, and set

$$
I_{A, K}=\bigcap_{i=1}^{n}\left(-\sqrt{K}+a_{i}, \sqrt{K}+a_{i}\right)=\left(-\sqrt{K}+a_{\max }, \sqrt{K}+a_{\min }\right) .
$$

The interval $I_{A, K}$ is not empty, i.e.

$$
a_{\max }-a_{\min }<2 \sqrt{K}
$$

For a fixed free vertical operator $A$ one can always obtain (1.4) by taking $K$ large enough. To understand the meaning of condition (III), note that $A$ can be diagonalized by a unitary transformation and the unperturbed operator $H_{0}$ can be rewritten as a direct sum of shifted Laplacians on the Bethe lattice (see [28]). It follows that the spectrum of $H_{0}$ is the union of the spectra of these shifted Laplacians, i.e. $\sigma\left(H_{0}\right)=\bigcup_{i=1}^{n}\left[-\sqrt{K}+a_{i}, \sqrt{K}+a_{i}\right]$. The interval $I_{A, K}$ is simply the interior of the intersection of the spectra of these shifted Laplacians, and condition (III) says that they all overlap.

Let us denote the standard basis elements of $\ell^{2}\left(\mathbb{B}, \mathbb{C}^{m}\right)$ by $|x, k\rangle$ for $x \in \mathbb{B}$ and $k \in\{1, \ldots, m\}$, i.e. $u=|x, k\rangle \in \ell^{2}\left(\mathbb{B}, \mathbb{C}^{m}\right)$ is the function $u(y)=\delta_{x, y} e_{k}$ where $e_{k}$ is the $k$-th standard basis vector of $\mathbb{C}^{m}$. A measure for the spread of a wave packet localized at $(x, j) \in \mathbb{B} \times \mathcal{I}$ is given by the square mean displacement

$$
r_{\lambda, x, j}^{2}(t):=\sum_{y \in \mathbb{B}} \sum_{k=1}^{m}[d((x, j),(y, k))]^{2}\left|\left\langle y, k\left|e^{-i t H_{\lambda}}\right| x, j\right\rangle\right|^{2},
$$

where $d((x, j),(y, k))$ denotes the distance between the sites $(x, j)$ and $(y, k)$. For an Anderson model on a product graph $\mathbb{B} \times \mathbb{G}$ this distance would be $d((x, j),(y, k))=$ $d(x, y)+d(j, k)$ where $d(j, k)$ is the distance between the vertices $j$ and $k$ on the graph $\mathbb{G}$. For a Wegner orbital model one would choose $d((x, j),(y, k))=d(x, y)$. In any case, we will use $d(x, y)$ as a lower bound. Ballistic motion means $r_{\lambda, x, j}^{2}(t) \sim$ $C t^{2}$, whereas diffusive behavior means $r_{\lambda, x, j}^{2}(t) \sim C t$ for large $t$. One always has ballistic motion as an upper bound,

$$
r_{\lambda, x, j}^{2}(t) \leq C t^{2}
$$


for some constant $C$ not depending on $x$ and $j$.

In order to show ballistic motion at least for some $|x, j\rangle$ we will consider the sum over $j$ at some arbitrary site of $\mathbb{B}$ which we will call the origin and denote by 0 . Furthermore we set $|x|=d(0, x)$ (which is not a norm) for $x \in \mathbb{B}$ and define

$$
r_{\lambda}^{2}(t):=\sum_{x \in \mathbb{B}} \sum_{j, k=1}^{m}|x|^{2}\left|\left\langle x, k\left|e^{-i t H_{\lambda}}\right| 0, j\right\rangle\right|^{2} .
$$

Note that

$$
\sum_{j=1}^{m} r_{\lambda, 0, j}^{2}(t) \geq r_{\lambda}^{2}(t)
$$

Theorem 1.1. For sufficiently small $\lambda$ we have

$$
\liminf _{t \rightarrow \infty} \frac{1}{t^{3}} \int_{0}^{t} \mathbb{E}\left(r_{\lambda}^{2}(s)\right) d s>0 .
$$

In particular, this implies

$$
\limsup _{t \rightarrow \infty} \mathbb{E}\left(\frac{r_{\lambda}^{2}(t)}{t^{2}}\right)>0 \quad \text { and } \mathbb{P}\left(\limsup _{t \rightarrow \infty} \frac{r_{\lambda}^{2}(t)}{t^{2}}>0\right)>0,
$$

and hence it follows from ergodicity that

$$
\mathbb{P}\left(\limsup _{t \rightarrow \infty} \frac{r_{\lambda, x, j}^{2}(t)}{t^{2}}>0 \text { for some }(x, j) \in \mathbb{B} \times I\right)=1 .
$$

We only need to prove (1.6), since (1.7) and (1.8) are consequences of (1.6) and (1.5) as shown in [24]. To prove (1.6), we start by reformulating the problem in terms of the matrix valued Green's function.

Given $x, y \in \mathbb{B}, z=E+i \eta$ with $E \in \mathbb{R}$ and $\eta>0$, the matrix valued Green's function $G_{\lambda}(x, y ; z)$ is the $m \times m$ matrix with entries

$$
\left[G_{\lambda}(x, y ; z)\right]_{j, k}:=\left\langle x, j\left|\left(H_{\lambda}-z\right)^{-1}\right| y, k\right\rangle .
$$

Using the spectral theorem and Plancherel's theorem, as in [24], Lemma A.2, we obtain

$$
\begin{aligned}
& \int_{0}^{\infty} e^{-\eta t} \mathbb{E}\left(r_{\lambda}^{2}(t)\right) d t \\
& \quad=\frac{1}{2 \pi} \int_{-\infty}^{\infty}\left\{\sum_{x \in \mathbb{B}}|x|^{2} \mathbb{E}\left(\operatorname{Tr}\left(\left|G_{\lambda}\left(0, x ; E+i \frac{\eta}{2}\right)\right|^{2}\right)\right)\right\} d E .
\end{aligned}
$$

Similarly to [24], eq. (2.3), we also have

$$
\int_{-\infty}^{\infty}\left\{\sum_{x \in \mathbb{B}}|x|^{2} \mathbb{E}\left(\operatorname{Tr}\left(\left|G_{\lambda}\left(0, x ; E+i \frac{\eta}{2}\right)\right|^{2}\right)\right)\right\} d E \leq \frac{4 \pi m^{2}}{\eta^{3}}\left\|\frac{1}{2} \Delta\right\|^{2} .
$$


In view of (1.9) and (1.10), Theorem 1.1 is a consequence of the following theorem using the Tauberian Theorem given in [33], Theorem 10.3. (Note that the proof is also valid for lim inf.)

Theorem 1.2. For sufficiently small $\lambda$ we have

$$
\liminf _{\eta \downarrow 0} \eta^{3} \int_{-\infty}^{\infty}\left\{\sum_{x \in \mathbb{B}}|x|^{2} \mathbb{E}\left(\operatorname{Tr}\left(\left|G_{\lambda}(0, x ; E+i \eta)\right|^{2}\right)\right)\right\} d E>0 .
$$

More precisely, there exists $\lambda_{0}>0$, such that for any $\lambda$ with $|\lambda|<\lambda_{0}$ we can find energies $E_{\lambda}^{ \pm} \in I_{A, K}=\left(-\sqrt{K}+a_{\max }, \sqrt{K}+a_{\min }\right)$, with $\lim _{\lambda \rightarrow 0} E_{\lambda}^{-}=-\sqrt{K}+a_{\max }$ and $\lim _{\lambda \rightarrow 0} E_{\lambda}^{+}=+\sqrt{K}+a_{\min }$, such that $H_{\lambda}$ has purely absolutely continuous spectrum in the interval $I_{\lambda}=\left(E_{\lambda}^{-}, E_{\lambda}^{+}\right)$, and

$$
\liminf _{\eta \downarrow 0} \eta^{3} \sum_{x \in \mathbb{B}}|x|^{2} \mathbb{E}\left(\left|G_{\lambda}(0, x ; E+i \eta)\right|^{2}\right)>0 \quad \text { for all } E \in I_{\lambda} .
$$

Eq. (1.11) follows immediately from (1.2) by Fatou's lemma. The fact that we find purely absolutely continuous spectrum for small $\lambda$ was proved in [28], so we only have to prove (1.2).

The paper is organized as follows. In Section 2 we introduce the basic supersymmetric formalism, reviewing the definitions and notation we used in [28]. Section 3 contains the new supersymmetric identities required for this work; see Theorem 3.3 and Corollary 3.4. In Section 4 we use these identities to rewrite the trace of the averaged matrix valued Green's function, $\mathbb{E}\left(\operatorname{Tr}\left(\left|G_{\lambda}(0, x ; z)\right|^{2}\right)\right)$, in a convenient form; see Proposition 4.3. We then finish the proof of Theorem 1.2 in Section 5.

\section{Supersymmetric methods}

The supersymmetric formalism described in this section can be found in more detail in [6], [11], [20], [29], [26], and [28]. We review the definitions and notation (we mostly use the same notation as in [28]) for the reader's convenience.

2.1. Basic definitions. $\operatorname{By}\left\{\psi_{k, \ell}, \bar{\psi}_{k, \ell} ; k=1, \ldots, m, \ell=1, \ldots, n\right\}$, where $m, n \in$ $\mathbb{N}$, we denote $2 m n$ independent Grassmann variables. They all anti-commute and are the generators of a Grassmann algebra isomorphic to $\Lambda^{2 m n}(\mathbb{R})$, given by the free algebra over $\mathbb{R}$ generated by these symbols modulo the ideal generated by the anti-commutators

$$
\psi_{i, j} \psi_{k, \ell}+\psi_{k, \ell} \psi_{i, j}, \quad \bar{\psi}_{i, j} \bar{\psi}_{k, \ell}+\bar{\psi}_{k, \ell} \bar{\psi}_{i, j}, \quad \bar{\psi}_{i, j} \psi_{k, \ell}+\psi_{k, \ell} \bar{\psi}_{i, j},
$$

where $i, k=1, \ldots, m$ and $j, \ell=1, \ldots, n$. This finite dimensional algebra will be denoted by $\Lambda(\boldsymbol{\Psi})$, where $\boldsymbol{\Psi}=\left(\bar{\psi}_{k, \ell}, \psi_{k, \ell}\right)_{k, \ell}$. The subset of one forms (linear combinations of the generators) is $\Lambda^{1}(\Psi)$. The complexification of $\Lambda(\Psi)$ is 
$\Lambda_{\mathbb{C}}(\Psi)=\mathbb{C} \otimes_{\mathbb{R}} \Lambda(\Psi)$. Sometimes we will also add and multiply expressions from different Grassmann algebras $\Lambda(\Psi)$ and $\Lambda\left(\Psi^{\prime}\right)$; these are to be understood as sums and products in the Grassmann algebra $\Lambda\left(\boldsymbol{\Psi}, \boldsymbol{\Psi}^{\prime}\right)$, generated by the entries of $\boldsymbol{\Psi}$ and $\boldsymbol{\Psi}^{\prime}$ as independent Grassmann variables.

A supervariable is an element of $\mathbb{R}^{2} \times \Lambda^{1}(\boldsymbol{\Psi}) \times \Lambda^{1}(\boldsymbol{\Psi})$. We introduce variables $\varphi_{k, \ell} \in \mathbb{R}^{2}$ and consider the supervariables $\varphi_{k, \ell}=\left(\varphi_{k, \ell}, \bar{\psi}_{k, \ell}, \psi_{k, \ell}\right)$. The collection $\boldsymbol{\Phi}=\left(\varphi_{k, \ell}\right)_{k, \ell}$ will be called an $m \times n$ supermatrix. More generally, an $m \times n$ matrix $\tilde{\boldsymbol{\Phi}}=\left(\tilde{\varphi}_{k, \ell}, \overline{\tilde{\psi}}_{k, \ell}, \tilde{\psi}_{k, \ell}\right)_{k, \ell} \in\left[\mathbb{R}^{2} \times \Lambda^{1}(\boldsymbol{\Psi}) \times \Lambda^{1}(\boldsymbol{\Psi})\right]^{m \times n}$ will be called a supermatrix if all the appearing one forms $\bar{\psi}_{k, \ell}, \tilde{\psi}_{k, \ell}, k=1,2 \ldots, m$ and $\ell=$ $1,2 \ldots, n$, are linearly independent. Supermatrices $\left(\boldsymbol{\Phi}_{i}\right)_{i}$ are said to be independent if $\boldsymbol{\Phi}_{i} \in \mathscr{L}_{m, n}\left(\boldsymbol{\Psi}_{i}\right)$ for all $i$, and all the entries of the different $\boldsymbol{\Psi}_{i}$ are independent Grassmann variables.

The collection of all supermatrices is a dense open subset of the vector space $\left[\mathbb{R}^{2} \times \Lambda^{1}(\Psi) \times \Lambda^{1}(\Psi)\right]^{m \times n}$ and will be denoted by $\mathscr{L}_{m, n}(\boldsymbol{\Psi})$, or just $\mathscr{L}_{m, n}$. Linear maps defined on $\mathscr{L}_{m, n}(\Psi)$ have to be understood as restrictions of linear maps defined on $\left[\mathbb{R}^{2} \times \Lambda^{1}(\boldsymbol{\Psi}) \times \Lambda^{1}(\boldsymbol{\Psi})\right]^{m \times n}$.

We also consider matrices $\varphi=\left(\varphi_{k, \ell}\right)_{k, \ell}$ with entries in $\mathbb{R}^{2}$. Writing each entry $\varphi_{k, \ell}$ as a row vector, $\varphi$ may be considered as $m \times 2 n$ matrix with real entries. Similarly, one may consider $\Psi$ as $m \times 2 n$ matrix with entries in $\Lambda^{1}(\Psi)$. With all these notations one may write $\Phi=(\varphi, \Psi)$, splitting a supermatrix into its real and Grassmannvariables parts.

For supervariables $\varphi_{1}=\left(\varphi_{1}, \bar{\psi}_{1}, \psi_{1}\right)$ and $\varphi_{2}=\left(\varphi_{2}, \bar{\psi}_{2}, \psi_{2}\right)$ we define

$$
\varphi_{1} \cdot \varphi_{2}:=\varphi_{1} \cdot \varphi_{2}+\frac{1}{2}\left(\bar{\psi}_{1} \psi_{2}+\bar{\psi}_{2} \psi_{1}\right) \text {. }
$$

For a supermatrix $\boldsymbol{\Phi}, \Phi_{k}=\left(\varphi_{k, \ell}\right)_{\ell=1 \ldots, n}$ denotes its $k$-th row vector. Given two supermatrices $\boldsymbol{\Phi}$ and $\boldsymbol{\Phi}^{\prime}$, we set

$$
\begin{aligned}
\Phi_{j}^{\prime} \cdot \Phi_{k} & :=\sum_{\ell=1}^{n} \varphi_{j, \ell}^{\prime} \cdot \varphi_{k, \ell} \quad \text { for } j, k=1,2, \ldots, m, \\
\boldsymbol{\Phi}^{\prime} \cdot \boldsymbol{\Phi} & :=\sum_{k=1}^{m} \Phi_{k}^{\prime} \cdot \Phi_{k}=\sum_{k=1}^{m} \sum_{\ell=1}^{n} \varphi_{k, \ell}^{\prime} \cdot \varphi_{k, \ell} .
\end{aligned}
$$

Given a supermatrix $\boldsymbol{\Phi}=(\boldsymbol{\varphi}, \boldsymbol{\Psi})$, where $\boldsymbol{\varphi} \in \mathbb{R}^{m \times 2 n}$ and $\boldsymbol{\Psi} \in \Lambda^{1}(\boldsymbol{\Psi})^{m \times 2 n}$, we introduce the $m \times m$ matrix $\boldsymbol{\Phi}^{\odot 2}$ with entries in $\Lambda(\Psi)$ by

$$
\begin{aligned}
& \left(\Phi^{\odot 2}\right)_{j, k}:=\Phi_{j} \cdot \Phi_{k}=\sum_{\ell=1}^{n}\left\{\varphi_{j, \ell} \cdot \varphi_{k, \ell}+\frac{1}{2}\left(\bar{\psi}_{j, \ell} \psi_{k, \ell}+\bar{\psi}_{k, \ell} \psi_{j, \ell}\right)\right\} \\
& =\sum_{\ell=1}^{n}\left\{\varphi_{j, \ell} \cdot \varphi_{k, \ell}+\left[\bar{\psi}_{j, \ell} \quad \psi_{j, \ell}\right]\left[\begin{array}{cc}
0 & 1 / 2 \\
-1 / 2 & 0
\end{array}\right]\left[\begin{array}{l}
\bar{\psi}_{k, \ell} \\
\psi_{k, \ell}
\end{array}\right]\right\} .
\end{aligned}
$$

It follows that

$$
\boldsymbol{\Phi}^{\odot 2}=\varphi^{\odot 2}+\boldsymbol{\Psi}^{\odot 2}, \quad \text { with } \varphi^{\odot 2}:=\varphi \varphi^{\top} \text { and } \boldsymbol{\Psi}^{\odot 2}:=\boldsymbol{\Psi} J \boldsymbol{\Psi}^{\top},
$$


where $J$ is the $2 n \times 2 n$ matrix consisting of $n$ blocks $\left[\begin{array}{cc}0 & 1 / 2 \\ -1 / 2 & 0\end{array}\right]$ along the diagonal. Note that $\Upsilon^{\top}$ will always denote the transpose of the matrix $\Upsilon$, whose entries may be elements of a Grassmann algebra.

Given a complex $m \times m$ matrix $B$, supermatrices $\boldsymbol{\Phi}, \boldsymbol{\Phi}^{\prime}$, and matrices $\boldsymbol{\varphi}^{\prime}, \boldsymbol{\varphi} \in$ $\mathbb{R}^{m \times 2 n}$, we define

$$
\begin{aligned}
\boldsymbol{\Phi}^{\prime} \cdot B \boldsymbol{\Phi} & :=\sum_{j, k=1}^{m} \sum_{\ell=1}^{n} B_{j, k} \varphi_{j, \ell}^{\prime} \cdot \varphi_{k, \ell}=\sum_{j, k=1}^{m} B_{j, k} \Phi_{j}^{\prime} \cdot \Phi_{k} \in \Lambda_{\mathbb{C}}(\Psi), \\
\boldsymbol{\varphi}^{\prime} \cdot B \boldsymbol{\varphi} & :=\sum_{j, k=1}^{n} \sum_{\ell=1}^{n} B_{j, k} \varphi_{j, \ell}^{\prime} \cdot \varphi_{k, \ell}=\operatorname{Tr}\left(\left(\boldsymbol{\varphi}^{\prime}\right)^{\top} B \boldsymbol{\varphi}\right) \in \mathbb{C} .
\end{aligned}
$$

Note that $\boldsymbol{\Phi} \cdot B \boldsymbol{\Phi}=\operatorname{Tr}\left(B \boldsymbol{\Phi}^{\odot 2}\right)$.

These definitions may be memorized as follows: If $n=1, \boldsymbol{\Phi}$ is a column vector indexed by $k, B \boldsymbol{\Phi}$ is the matrix vector product, and $\boldsymbol{\Phi}^{\prime} \cdot B \boldsymbol{\Phi}$ is the dot product of vectors of supervariables. For general $n$ the supermatrix $\boldsymbol{\Phi}$ has columns indexed by $\ell=1,2, \ldots, n$, "the $n$ replicas", and in all definitions of dot products there is an additional sum over this index.

A complex superfunction with respect to $\Lambda(\Psi)$ is a function $F: \mathbb{R}^{m \times 2 n} \rightarrow \Lambda_{\mathbb{C}}(\Psi)$. Let $\beta_{i} \in \Lambda(\Psi)$ for $i \in\left\{1, \ldots, 2^{2 m n}\right\}$ be a basis for $\Lambda(\Psi)$ over $\mathbb{R}$. Each $\beta_{i}$ is a polynomial in the entries of $\boldsymbol{\Psi}$ (we required the entries of $\boldsymbol{\Psi}$ to be independent) and $F$ is of the form

$$
F(\varphi)=\sum_{i=1}^{2 m n} F_{i}(\varphi) \beta_{i}, \quad \text { where } F_{i}: \mathbb{R}^{m \times 2 n} \rightarrow \mathbb{C} .
$$

We interpret this as a function $F(\boldsymbol{\Phi})$ where $\boldsymbol{\Phi}=(\boldsymbol{\varphi}, \boldsymbol{\Psi})$. In this sense the map $\boldsymbol{\Phi} \mapsto \operatorname{Tr}\left(B \boldsymbol{\Phi}^{\odot 2}\right)$, where $B$ is a complex $m \times m$ matrix, is a superfunction. Similarly, we can define superfunctions $F\left(\boldsymbol{\Phi}_{1}, \ldots, \boldsymbol{\Phi}_{k}\right)$ of $k$ independent supermatrices using the Grassmann algebra $\Lambda\left(\left(\boldsymbol{\Psi}_{j}\right)_{j \in\{1, \ldots, k\}}\right)$. We write $F \in S\left(\mathscr{L}_{m, n}\right)$, or $F \in C^{\infty}\left(\mathscr{L}_{m, n}\right)$, if for all $i$ we have $F_{i} \in S\left(\mathbb{R}^{m \times 2 n}\right)$, the Schwartz space, or $F_{i} \in C^{\infty}\left(\mathbb{R}^{m \times 2 n}\right)$, respectively.

We define the integral over the Grassmann variables in the following way. For a fixed pair $k, \ell$ we write $F=F(\boldsymbol{\Phi})$ as $F=F_{0}^{k, \ell}+F_{1}^{k, \ell} \bar{\psi}_{k, \ell}+F_{2}^{k, \ell} \psi_{k, \ell}+$ $F_{3}^{k, \ell} \bar{\psi}_{k, \ell} \psi_{k, \ell}$ where the $F_{i}^{k, \ell}$ are superfunctions not depending on $\bar{\psi}_{k, \ell}$ and $\psi_{k, \ell}$. Then

$$
\int F d \bar{\psi}_{k, \ell} d \psi_{k, \ell}:=-F_{3}^{k, \ell}
$$

If all functions $F_{i}$ in the expansion (2.8) are in $L^{1}\left(\mathbb{R}^{m \times 2 n}\right)$, we say that $F \in L^{1}\left(\mathscr{L}_{m, n}\right)$ and define the supersymmetric integral by

$$
\int F(\boldsymbol{\Phi}) D \boldsymbol{\Phi}=\frac{1}{\pi^{m n}} \int F(\boldsymbol{\Phi}) \prod_{k=1}^{m} \prod_{\ell=1}^{n} d^{2} \varphi_{k, \ell} d \bar{\psi}_{k, \ell} d \psi_{k, \ell}
$$


2.2. Differential operators and supersymmetric functions. We now recall the notion of smooth supersymmetric functions and introduce certain differential operators; we refer to [28] for details. We will use the notation of [28] except for some small sign deviations that will be explicitly pointed out.

We start by introducing convenient notation for Grassmann monomials. Recall $\mathcal{I}=\{1, \ldots, m\}$; we will denote the set of subsets of $\mathcal{I}$ by $\mathfrak{P}(\mathcal{I})$. Given $(\bar{a}, a) \in$ $(\mathfrak{P}(\mathcal{I}))^{2}=\mathfrak{P}(\mathcal{I}) \times \mathfrak{P}(\mathcal{I})$ and $\bar{a}=\left\{\bar{k}_{1}, \ldots, \bar{k}_{c}\right\}, a=\left\{k_{1}, \ldots, k_{d}\right\}$, both ordered (i.e. $\bar{k}_{i}<\bar{k}_{j}$ and $k_{i}<k_{j}$ if $i<j$ ), we set

$$
\Psi_{\bar{a}, a, \ell}:=\left(\prod_{j=1}^{|\bar{a}|} \bar{\psi}_{\bar{k}_{j}, \ell}\right)\left(\prod_{j=1}^{|a|} \psi_{k_{j}, \ell}\right),
$$

using the conventions $\prod_{j=1}^{c} \psi_{j}=\psi_{1} \psi_{2} \cdots \psi_{c}$ for non-commutative products and $\prod_{j=1}^{0} \psi_{j}=1$. In particular, $\Psi_{\emptyset, \emptyset, \ell}=1$.

An important subset of $(\mathfrak{P}(\mathcal{I}))^{2}$ is $\mathcal{P}$, the set of pairs $(\bar{a}, a)$ of subsets of $\mathcal{I}$ with the same cardinality, i.e.

$$
\mathcal{P}=\{(\bar{a}, a): \bar{a}, a \subset \mathcal{I},|\bar{a}|=|a|\} .
$$

More generally, for each $k \in[-m, m] \cap \mathbb{Z}$ we define

$$
\mathcal{P}_{k}:=\{(\bar{a}, a): \bar{a}, a \subset \mathcal{I},|\bar{a}|=|a|+k\},
$$

so, in particular, $\mathcal{P}=\mathcal{P}_{0}$. (A note of caution: we used $\mathcal{P}_{m}$ for $\mathcal{P}=\mathcal{P}_{0}$ in [28].)

For $(\bar{a}, a) \in \mathcal{P}$ we set

$$
\Psi_{\bar{a}, a}^{(\ell)}:=\prod_{j=1}^{|a|}\left(\bar{\psi}_{\bar{k}_{j}, \ell} \psi_{k_{j}, \ell}\right), \quad \text { with the convention } \Psi_{\emptyset, \emptyset}^{(\ell)}:=1 .
$$

Note that these Grassmann monomials are slightly different from the ones we defined in (2.10). In fact, counting transpositions we get

$$
\Psi_{\bar{a}, a, \ell}=(-1)^{\frac{|a||| a \mid-1)}{2}} \Psi_{\bar{a}, a}^{(\ell)} \quad \text { for }(\bar{a}, a) \in \mathcal{P} .
$$

Given a pair of $n$-tuples of subsets of $\mathcal{I},(\overline{\boldsymbol{a}}, \boldsymbol{a}) \in(\mathfrak{P}(\mathcal{I}))^{n} \times(\mathfrak{P}(\mathcal{I}))^{n}$ with $\overline{\boldsymbol{a}}=$ $\left(\bar{a}_{1}, \ldots, \bar{a}_{n}\right)$ and $\boldsymbol{a}=\left(a_{1}, \ldots, a_{n}\right)$, we set

$$
\Psi_{\bar{a}, \boldsymbol{a}}:=\prod_{\ell=1}^{n} \Psi_{\bar{a}_{\ell}, a_{\ell}, \ell} .
$$

An important subset of $(\mathfrak{P}(\mathcal{I}))^{n} \times(\mathfrak{P}(\mathcal{I}))^{n}$ is given by the set

$$
\mathcal{P}^{n}:=\left\{(\overline{\boldsymbol{a}}, \boldsymbol{a}) \in(\mathfrak{P}(\mathcal{I}))^{n} \times(\mathfrak{P}(\mathcal{I}))^{n}:\left(\bar{a}_{\ell}, a_{\ell}\right) \in \mathcal{P} \text { for } \ell=1, \ldots, n\right\} .
$$


This set is canonically isomorphic to the Cartesian product $(\mathcal{P})^{\times n}$, justifying the notation. However, we will use $n$ as an upper index to indicate that we deal with $n$-tuples of sets, and, given $k \in[-m, m] \cap \mathbb{Z}$, set

$$
\begin{aligned}
\mathcal{P}_{k}^{n}:=\left\{(\overline{\boldsymbol{a}}, \boldsymbol{a}) \in(\mathfrak{P}(\mathcal{I}))^{n} \times(\mathfrak{P}(\mathcal{I}))^{n}:\right. & \\
& \left.\left(\bar{a}_{1}, a_{1}\right) \in \mathcal{P}_{k},\left(\bar{a}_{\ell}, a_{\ell}\right) \in \mathcal{P} \text { for } \ell=2, \ldots, n\right\} .
\end{aligned}
$$

If $(\overline{\boldsymbol{a}}, \boldsymbol{a}) \in \mathcal{P}_{k}^{n}$ the sets $\bar{a}_{\ell}$ and $a_{\ell}$ have the same cardinality except for possibly $\ell=1$, where $\left|\bar{a}_{1}\right|=\left|a_{1}\right|+k$. Note that $\mathcal{P}_{0}^{n}=\mathcal{P}^{n}$. In view of (2.12), for $\boldsymbol{a} \in(\mathfrak{P}(\mathcal{I}))^{n}$ we define

$$
\operatorname{sgn}(\boldsymbol{a})=\prod_{\ell=1}^{n}(-1)^{\frac{\left|a_{\ell}\right|\left(\left|a_{\ell}\right|-1\right)}{2}},
$$

getting

$$
\prod_{\ell=1}^{n} \Psi_{\bar{a}_{\ell}, a_{\ell}}^{(\ell)}=\operatorname{sgn}(\boldsymbol{a}) \Psi_{\overline{\boldsymbol{a}}, \boldsymbol{a}} \quad \text { for }(\overline{\boldsymbol{a}}, \boldsymbol{a}) \in \mathcal{P}^{n} .
$$

(Note: the left hand side of (2.14) corresponds to the definition of $\Psi_{\overline{\boldsymbol{a}}, \boldsymbol{a}}$ for $(\overline{\boldsymbol{a}}, \boldsymbol{a}) \in$ $\mathcal{P}^{n}$ in [28], eq. (2.25).)

The counterpart to these Grassmann monomials are differential operators acting on functions defined on $\mathrm{Sym}^{+}(m)$, the space of non-negative, real, symmetric $m \times m$ matrices. From now on we assume $n \geq \frac{m}{2}$, so that the map $\varphi \in \mathbb{R}^{m \times 2 n} \mapsto \varphi^{\odot 2}=$ $\boldsymbol{\varphi} \varphi^{t} \in \mathrm{Sym}^{+}(m)$ is surjective.

Let $C^{\infty}\left(\operatorname{Sym}^{+}(m)\right)$ denote the set of continuous functions $f$ on $\operatorname{Sym}^{+}(m)$ which are $C^{\infty}$ on the interior of $\operatorname{Sym}^{+}(m)$. We let $\partial_{j, k}$ denote the partial derivative with respect to the $j, k$-entry of the symmetric matrix, i.e. $\partial_{j, k} f(M)=\frac{\partial}{\partial M_{k, j}} f(M)$ for $f \in C^{\infty}\left(\operatorname{Sym}^{+}(m)\right)$. Note that $\partial_{j, k}=\partial_{k, j}$. We also set $\tilde{\partial}_{j, k}=\frac{1}{2} \partial_{j, k}$ for $j \neq k$ and $\tilde{\partial}_{j, j}=\partial_{j, j}$.

Given $(\bar{a}, a) \in \mathcal{P}$ with $a \neq \emptyset, \bar{a}=\left\{\bar{k}_{1}, \ldots, \bar{k}_{c}\right\}$ and $a=\left\{k_{1}, \ldots, k_{c}\right\}$, both ordered, we define the matrix-differential operator

$$
\boldsymbol{\partial}_{\bar{a}, a}:=\left(\begin{array}{ccc}
\tilde{\partial}_{\bar{k}_{1}, k_{1}} & \cdots & \tilde{\partial}_{\bar{k}_{1}, k_{c}} \\
\vdots & \ddots & \vdots \\
\tilde{\partial}_{\bar{k}_{c}, k_{1}} & \cdots & \tilde{\partial}_{\bar{k}_{c}, k_{c}}
\end{array}\right) .
$$

Furthermore we set $D_{\emptyset, \emptyset}$ to be the identity operator and

$$
D_{\bar{a}, a}:=\operatorname{det}\left(\partial_{\bar{a}, a}\right) \quad \text { if } a \neq \emptyset .
$$

Note that $\partial_{\bar{a}, a}=\partial_{a, \bar{a}}^{\top}$, and hence $D_{\bar{a}, a}=D_{a, \bar{a}}$. In the special case when $\bar{a}=a=\mathcal{I}$, we set

$$
\boldsymbol{\partial}:=\boldsymbol{\partial}_{\mathcal{I}, \mathcal{I}} \quad \text { and } \delta:=\operatorname{det}(\boldsymbol{\partial})=D_{\mathcal{I}, \mathcal{I}} .
$$


Furthermore, for $(\bar{a}, a) \in \mathcal{P}$ we set $\left(\bar{a}^{\mathrm{c}}, a^{\mathrm{c}}\right):=(\mathcal{I} \backslash \bar{a}, \mathcal{I} \backslash a) \in \mathcal{P}$. Note that the $(j, k)$-entry of $\boldsymbol{\partial}$ is $\boldsymbol{\partial}_{\{j\},\{k\}}=D_{\{j\},\{k\}}$. The cofactor is given by $(-1)^{j+k} D_{\{j\}}$ c $\left.\{k\}\right\}^{\mathrm{c}}$. The transpose of the cofactor matrix of $\partial$ will be denoted by $\boldsymbol{\phi}$, i.e.

$$
\begin{aligned}
\mathscr{d} & :=\left((-1)^{j+k} D_{\{k\}^{\mathrm{c}},\{j\}^{\mathrm{c}}}\right)_{j, k \in \mathcal{I}} \\
& =\left(\begin{array}{cccc}
D_{\{1\}^{\mathrm{c}},\{1\}^{\mathrm{c}}} & -D_{\{2\}^{\mathrm{c}},\{1\}^{\mathrm{c}}} & \ldots & (-1)^{m+1} D_{\{m\}^{\mathrm{c}},\{1\}^{\mathrm{c}}} \\
-D_{\{1\}^{\mathrm{c}},\{2\}^{\mathrm{c}}} & D_{\{2\}^{\mathrm{c}},\{2\}^{\mathrm{c}}} & \ldots & (-1)^{m} D_{\{m\}^{\mathrm{c}},\{2\}^{\mathrm{c}}} \\
\vdots & \vdots & \ddots & \vdots \\
(-1)^{m+1} D_{\{1\}^{\mathrm{c}},\{m\}^{\mathrm{c}}} & (-1)^{m} D_{\{2\}^{\mathrm{c}},\{m\}^{\mathrm{c}}} & \ldots & D_{\{m\}^{\mathrm{c}},\{m\}^{\mathrm{c}}}
\end{array}\right) .
\end{aligned}
$$

With these definitions we have

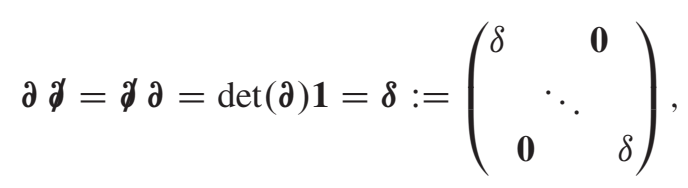

where $\mathbf{1}=\mathbf{1}_{m}$ denotes the unit $m \times m$ matrix and $\boldsymbol{\delta}$ is a diagonal $m \times m$ matrix with $\delta$ on all diagonal entries. Furthermore, for $(\overline{\boldsymbol{a}}, \boldsymbol{a}) \in \mathcal{P}^{n}$ we set

$$
D_{\bar{a}, \boldsymbol{a}}:=\prod_{\ell=1}^{n} D_{\bar{a}_{\ell}, a_{\ell}} .
$$

We have $D_{\overline{\boldsymbol{a}} \boldsymbol{a}}=D_{\boldsymbol{a}, \overline{\boldsymbol{a}}}$. For $f \in C^{\infty}\left(\operatorname{Sym}^{+}(m)\right)$ and $\operatorname{det}\left(\varphi^{\odot 2}\right) \neq 0$, a formal Taylor expansion yields (cf [28], eq. (2.26))

$$
f\left(\boldsymbol{\Phi}^{\odot 2}\right)=\sum_{(\bar{a}, \boldsymbol{a}) \in \mathcal{P} n} D_{\overline{\boldsymbol{a}}, \boldsymbol{a}} f\left(\boldsymbol{\varphi}^{\odot 2}\right) \operatorname{sgn}(\boldsymbol{a}) \Psi_{\overline{\boldsymbol{a}}, \boldsymbol{a}} .
$$

Let $C_{n}^{\infty}\left(\operatorname{Sym}^{+}(m)\right)$ denote the set of all functions $f \in C^{\infty}\left(\operatorname{Sym}^{+}(m)\right)$ where $\varphi \mapsto D_{\bar{a}, \boldsymbol{a}} f\left(\varphi^{\odot 2}\right)$, defined on the dense open set where $\operatorname{det}\left(\varphi^{\odot 2}\right) \neq 0$, extends (uniquely) to a $C^{\infty}$ function on $\mathbb{R}^{m \times 2 n}$ for all $(\overline{\boldsymbol{a}}, \boldsymbol{a}) \in \mathcal{P}^{n}$.

Definition 2.1. Let $n \geq \frac{m}{2}$.

(i) The set $S C^{\infty}\left(\mathscr{L}_{m, n}\right)$ of smooth supersymmetric functions is defined as the set of all smooth superfunctions $F(\boldsymbol{\Phi})$ such that $F(\boldsymbol{\Phi})=f\left(\boldsymbol{\Phi}^{\odot 2}\right)$ for some $f \in$ $C_{n}^{\infty}\left(\operatorname{Sym}^{+}(m)\right)$.

(ii) The set $S S\left(\mathscr{L}_{m, n}\right):=S\left(\mathscr{L}_{m, n}\right) \cap S C^{\infty}\left(\mathscr{L}_{m, n}\right)$ denotes the supersymmetric Schwartz functions.

This definition is justified by [28], Proposition 2.3, which is based on [29], Corollary 2.9. $S C^{\infty}\left(\mathscr{L}_{m, n}\right)$ can be identified with $C_{n}^{\infty}\left(\operatorname{Sym}^{+}(m)\right)$. Furthermore, if we define the subset $S_{n}\left(\operatorname{Sym}^{+}(m)\right)$ of all functions $f \in C_{n}^{\infty}\left(\operatorname{Sym}^{+}(m)\right)$ where $\varphi \mapsto D_{\bar{a}, \boldsymbol{a}} f\left(\varphi^{\odot 2}\right)$ is a Schwartz function, then $S S\left(\mathscr{L}_{m, n}\right)$ can be identified with $\oint_{n}\left(\operatorname{Sym}^{+}(m)\right)$. 
Finally let us define some algebraic operations on $(\mathfrak{P}(\mathcal{I}))^{n}$ which will give some Leibniz type formulas and will be useful later. Let $\boldsymbol{a}, \boldsymbol{b} \in(\mathfrak{P}(\mathcal{I}))^{n}$. If $a_{\ell} \cap b_{\ell}=\emptyset$ for each $\ell=1, \ldots, n$, then we say $\boldsymbol{a}$ and $\boldsymbol{b}$ are addable and define $\boldsymbol{c}=\boldsymbol{a}+\boldsymbol{b} \in(\mathfrak{P}(\mathcal{I}))^{n}$ by $c_{\ell}=a_{\ell} \cup b_{\ell}$. Similarly, if $b_{\ell} \subset a_{\ell}$ for all $\ell$, then we define $\boldsymbol{c}=\boldsymbol{a}-\boldsymbol{b}$ by $c_{\ell}=a_{\ell} \backslash b_{\ell}$. If $\boldsymbol{a}$ and $\boldsymbol{b}$ are addable, then $(\boldsymbol{a}+\boldsymbol{b})-\boldsymbol{b}=\boldsymbol{a}$. Furthermore we denote by $\mathcal{I}$ the $n$-tuple where each entry is the full set $\mathcal{I}, \mathcal{I}=(\mathcal{I}, \mathcal{I}, \ldots, I)$. Moreover, we define $\boldsymbol{a}^{\boldsymbol{c}}=\mathfrak{I}-\boldsymbol{a}$. We say that $(\overline{\boldsymbol{a}}, \boldsymbol{a})$ and $(\overline{\boldsymbol{b}}, \boldsymbol{b}) \in \mathfrak{P}(\mathcal{I})^{n} \times \mathfrak{P}(\mathcal{I})^{n}$ are addable if $\overline{\boldsymbol{a}}+\overline{\boldsymbol{b}}$ and $\boldsymbol{a}+\boldsymbol{b}$ are defined by the notion above. In this case we define $\operatorname{sgn}(\overline{\boldsymbol{a}}, \boldsymbol{a}, \overline{\boldsymbol{b}}, \boldsymbol{b}) \in\{-1,1\}$ by

$$
\Psi_{\bar{a}, \boldsymbol{a}} \Psi_{\overline{\boldsymbol{b}}, \boldsymbol{b}}=\operatorname{sgn}(\overline{\boldsymbol{a}}, \boldsymbol{a}, \overline{\boldsymbol{b}}, \boldsymbol{b}) \Psi_{\overline{\boldsymbol{a}}+\overline{\boldsymbol{b}}, \boldsymbol{a}+\boldsymbol{b}} .
$$

(Note of caution: this definition of $\operatorname{sgn}(\overline{\boldsymbol{a}}, \boldsymbol{a}, \overline{\boldsymbol{b}}, \boldsymbol{b})$ differs from [28], eq. (2.27), since our definition of $\Psi_{\overline{\boldsymbol{a}}, \boldsymbol{a}}$ in (2.13) is different from [28], eq. (2.25).) If $(\overline{\boldsymbol{a}}, \boldsymbol{a}) \in \mathcal{P}_{j}^{n}$ and $(\overline{\boldsymbol{b}}, \boldsymbol{b}) \in \mathcal{P}_{k}^{n}$ are addable, then $(\overline{\boldsymbol{a}}+\boldsymbol{a}, \overline{\boldsymbol{b}}+\boldsymbol{b}) \in \mathcal{P}_{j+k}^{n}$ and $\left(\overline{\boldsymbol{a}}^{\boldsymbol{c}}, \boldsymbol{a}^{\boldsymbol{c}}\right),(\boldsymbol{a}, \overline{\boldsymbol{a}}) \in \mathcal{P}_{-j}^{n}$.

Since the product of two supersymmetric functions is supersymmetric, for all $f, g \in C_{n}^{\infty}\left(\operatorname{Sym}^{+}(m)\right)$ and all $(\overline{\boldsymbol{a}}, \boldsymbol{a}) \in \mathcal{P}^{n}$ we have

$$
D_{\overline{\boldsymbol{a}}, \boldsymbol{a}}(f g)=\sum_{\substack{(\overline{\boldsymbol{b}}, \boldsymbol{b}),\left(\overline{\boldsymbol{b}}^{\prime}, \boldsymbol{b}^{\prime}\right) \in \mathcal{P}^{n} \\ \overline{\boldsymbol{b}}+\overline{\boldsymbol{b}}^{\prime}=\overline{\boldsymbol{a}}, \boldsymbol{b}+\boldsymbol{b}^{\prime}=\boldsymbol{a}}} \frac{\operatorname{sgn}(\boldsymbol{b}) \operatorname{sgn}\left(\boldsymbol{b}^{\prime}\right) \operatorname{sgn}(\boldsymbol{a})}{\operatorname{sgn}\left(\overline{\boldsymbol{b}}, \boldsymbol{b}, \overline{\boldsymbol{b}}^{\prime}, \boldsymbol{b}^{\prime}\right)} D_{\overline{\boldsymbol{b}}, \boldsymbol{b}} g D_{\overline{\boldsymbol{b}}^{\prime}, \boldsymbol{b}^{\prime}} f .
$$

2.3. The supersymmetric Fourier transform. We recall the definition of the supersymmetric Fourier transform. Given $f \in S S_{n}\left(\operatorname{Sym}^{+}(m)\right)$ we define $T f \in$ $S S_{n}\left(\operatorname{Sym}^{+}(m)\right)$ by

$$
(T f)\left(\left(\boldsymbol{\Phi}^{\prime}\right)^{\odot 2}\right)=\int e^{l \boldsymbol{\Phi}^{\prime} \cdot \boldsymbol{\Phi}} f\left(\boldsymbol{\Phi}^{\odot 2}\right) D \boldsymbol{\Phi},
$$

where we use the fact that the right hand side defines a supersymmetric function [29]. It follows that [28], eq. (2.37)

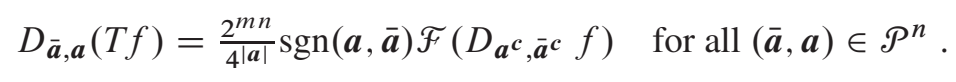

Here $\mathcal{F}$ denotes the Fourier transform on $\mathbb{R}^{m \times 2 n}$; we abuse the notation by letting $\mathcal{F} f$ denote the function in $S_{n}\left(\mathrm{Sym}^{+}(m)\right)$ such that $(\mathscr{F} f)\left(\varphi^{\odot 2}\right)$ is the Fourier transform of the function $F(\varphi)=f\left(\varphi^{\odot 2}\right)$. In addition,

$$
\begin{aligned}
\operatorname{sgn}(\overline{\boldsymbol{a}}, \boldsymbol{a}) & :=\int \operatorname{sgn}(\boldsymbol{a}) \Psi_{\overline{\boldsymbol{a}}, \boldsymbol{a}} \operatorname{sgn}\left(\boldsymbol{a}^{\boldsymbol{c}}\right) \Psi_{\overline{\boldsymbol{a}}^{c}, \boldsymbol{a}^{\boldsymbol{c}}} D \Psi \\
& =(-1)^{m n} \frac{\operatorname{sgn}(\boldsymbol{a}) \operatorname{sgn}\left(\boldsymbol{a}^{\boldsymbol{c}}\right) \operatorname{sgn}(\mathcal{I})}{\operatorname{sgn}\left(\overline{\boldsymbol{a}}, \boldsymbol{a}, \overline{\boldsymbol{a}}^{\boldsymbol{c}}, \boldsymbol{a}^{\boldsymbol{c}}\right)}
\end{aligned}
$$

for $(\overline{\boldsymbol{a}}, \boldsymbol{a}) \in \mathcal{P}^{n}$, where $D \boldsymbol{\Psi}=\prod_{k, \ell} d \bar{\psi}_{k, \ell} d \psi_{k, \ell}$. By (2.14) this is the same definition as [28], eq. (2.32); the second equality follows from (2.18) and

$$
\int \Psi_{\mathfrak{X}, \mathfrak{I}} D \Psi=(-1)^{m n} \operatorname{sgn}(\mathfrak{I}) .
$$


Moreover we have, as in [28], eq. (2.39),

$$
T^{2} f=T T f=f \quad \text { for all } f \in S_{n}\left(\operatorname{Sym}^{+}(m)\right) .
$$

In the special cases when $(\overline{\boldsymbol{a}}, \boldsymbol{a})=(\mathcal{I}, \mathcal{I})$ and $(\overline{\boldsymbol{a}}, \boldsymbol{a})=\left(\mathcal{I}^{\boldsymbol{c}}, \mathcal{I}^{\boldsymbol{c}}\right),(2.21)$ yields

$$
\delta^{n} T f=(-2)^{-m n} \mathcal{F} f \text { and } T f=(-2)^{m n} \mathcal{F} \delta^{n} f .
$$

In particular, we have

$$
\mathscr{F}=4^{m n} \delta^{n} \mathcal{F} \delta^{n} .
$$

An important special case of (2.21) is the following. For $k \in \mathcal{I}$ let

$$
\llbracket k \rrbracket:=(\{k\}, \emptyset, \ldots, \varnothing) \in \mathfrak{P}(\mathcal{I})^{n},
$$

then $\Psi_{\llbracket j \rrbracket, \llbracket k \rrbracket}=\bar{\psi}_{j, 1} \psi_{k, 1}, D_{\llbracket j \rrbracket, \llbracket k \rrbracket}=D_{\{j\},\{k\}}$ and $D_{\llbracket j \rrbracket^{c}, \llbracket k \rrbracket^{c}}=D_{\{k\}^{c},\{j\}^{c}} \delta^{n-1}$. Using the notation of (2.11) we get

$$
\bar{\psi}_{j, 1} \psi_{k, 1} \Psi_{\{j\}^{\mathrm{c}},\{k\}^{\mathrm{c}}}^{(1)}=(-1)^{j+k} \Psi_{\mathcal{I}, \mathcal{I}}^{(1)}
$$

since one needs $j-1$ transpositions to bring the $\bar{\psi}$ 's to order and $k-1$ transpositions to bring the $\psi$ 's to order. In view of (2.14) this implies

$$
\operatorname{sgn}(\llbracket j \rrbracket) \Psi_{\llbracket j \rrbracket, \llbracket k \rrbracket} \operatorname{sgn}\left(\llbracket j \rrbracket^{c}\right) \Psi_{\llbracket j \rrbracket^{c}, \llbracket k \rrbracket^{c}}=(-1)^{j+k} \operatorname{sgn}(\mathcal{I}) \Psi_{\mathfrak{I}, \boldsymbol{I}},
$$

which by (2.22) and (2.23) yields

$$
\operatorname{sgn}(\llbracket j \rrbracket, \llbracket k \rrbracket)=\int(-1)^{j+k} \operatorname{sgn}(\mathcal{I}) \Psi_{\mathfrak{L}, \mathfrak{I}} D \Psi=(-1)^{m n}(-1)^{j+k} .
$$

Thus, using $\overline{\boldsymbol{a}}=\llbracket j \rrbracket$ and $\boldsymbol{a}=\llbracket k \rrbracket$ in (2.21) gives

$$
D_{\{j\},\{k\}}(T f)=2^{m n-2}(-1)^{m n}(-1)^{j+k} \mathcal{F}\left(\delta^{n-1} D_{\{k\}^{\mathrm{c}},\{j\}^{\mathrm{c}}} f\right) .
$$

In particular, given $\boldsymbol{f}=\left(f_{1}, \ldots, f_{m}\right)^{\top} \in\left[S_{n}\left(\operatorname{Sym}^{+}(m)\right)\right]^{m \times 1}$, a column vector of elements of $S_{n}\left(\operatorname{Sym}^{+}(m)\right)$, setting

$$
T \boldsymbol{f}=\left(T f_{1}, \ldots, T f_{m}\right)^{\top} \text { and } \mathscr{F} \boldsymbol{f}=\left(\mathscr{F} f_{1}, \ldots, \mathcal{F} f_{m}\right)^{\top}
$$

we have

$$
\partial(T \boldsymbol{f})=(-2)^{m n-2} \mathcal{F}\left(\boldsymbol{\delta}^{n-1} \boldsymbol{\gamma} \boldsymbol{f}\right) .
$$

As in [28], following Campanino and Klein, [7], [20], and [29], we introduce norms $\|\cdot\|_{p}$ on $C_{n}^{\infty}\left(\operatorname{Sym}^{+}(m)\right)$, with $p \in[1, \infty]$, by

$$
\|f\|_{p}^{2}:=\sum_{(\overline{\boldsymbol{a}}, \boldsymbol{a}) \in \mathcal{P}^{n}}\left\|2^{|\boldsymbol{a}|} D_{\overline{\boldsymbol{a}}, \boldsymbol{a}} f\left(\boldsymbol{\varphi}^{\odot 2}\right)\right\|_{L^{p}\left(\mathbb{R}^{m \times 2 n}, d^{2 m n} \boldsymbol{\varphi}\right)}^{2} .
$$


We define the Hilbert space $\mathscr{H}$ as completion of $S_{n}\left(\operatorname{Sym}^{+}(m)\right)$ with respect to the norm $\|\cdot\|_{2}$. The Banach spaces $\mathscr{H}_{p}, p \in[1, \infty]$, are defined by

$$
\mathscr{H}_{p}:=\left\{f \in \mathscr{H}:\|f\|_{\mathscr{H}_{p}}:=\|f\|_{2}+\|f\|_{p}<\infty\right\} .
$$

We also define the Banach space $\tilde{\mathscr{H}}_{\infty}$ as the completion of

$$
\tilde{\mathcal{H}}_{\infty}^{(0)}:=\left\{f \in C_{n}^{\infty}\left(\operatorname{Sym}^{+}(m)\right):\|f\|_{\infty}<\infty\right\}
$$

with respect to the norm $\|\cdot\|_{\infty}$. In view of (2.21) and (2.27), as mentioned in [28], the supersymmetric Fourier transform $T$ extends to $\mathscr{H}$ as a unitary operator.

We also want to consider tensor products. For $g\left(\varphi_{+}^{\odot 2}, \varphi_{-}^{\odot 2}\right) \in C_{n}^{\infty}\left(\operatorname{Sym}^{+}(m)\right) \otimes$ $C_{n}^{\infty}\left(\operatorname{Sym}^{+}(m)\right)$ we define the tensor norms

$$
\|\| g\left\|_{p}^{2}=\sum_{\substack{(\overline{\boldsymbol{a}}, \boldsymbol{a}) \in \mathcal{P}^{n} \\(\overline{\boldsymbol{b}}, \boldsymbol{b}) \in \mathcal{P}^{n}}}\right\| 2^{|\boldsymbol{a}|+|\boldsymbol{b}|} D_{\overline{\boldsymbol{a}}, \boldsymbol{a}}^{(+)} D_{\overline{\boldsymbol{b}}, \boldsymbol{b}}^{(-)} g\left(\varphi_{+}^{\odot 2}, \boldsymbol{\varphi}_{-}^{\odot 2}\right) \|_{L^{p}\left(\boldsymbol{\varphi}_{+}, \boldsymbol{\varphi}_{-}\right)}^{2},
$$

where $\|\cdot\|_{L^{p}\left(\boldsymbol{\varphi}_{+}, \boldsymbol{\varphi}_{-}\right)}$denotes the $p$-norm of the $L^{p}$ space on $\left(\mathbb{R}^{m \times 2 n^{2}}\right.$ in the variables $\boldsymbol{\varphi}_{+}, \boldsymbol{\varphi}_{-}$with respect to the Lebesgue measure $d^{2 m n} \boldsymbol{\varphi}_{+} d^{2 m n} \boldsymbol{\varphi}_{-} . D_{\overline{\boldsymbol{a}}, \boldsymbol{a}}^{(+)}, D_{\overline{\boldsymbol{a}}, \boldsymbol{a}}^{(-)}$ denote the differential operator $D_{\bar{a}, \boldsymbol{a}}$ with respect to $\varphi_{+}^{\odot 2}$ and $\varphi_{-}^{\odot 2}$ respectively. The Hilbert space tensor product $\mathcal{K}:=\mathscr{H} \otimes \mathscr{H}$ is the completion of $S_{n}\left(\operatorname{Sym}^{+}(m)\right) \otimes$ $S_{n}\left(\operatorname{Sym}^{+}(m)\right)$ with respect to the norm \|\|$\cdot\|\|_{2}$. The unitary operator $T$ induces the unitary transformation

$$
\widehat{T}:=T \otimes T \text { on } \mathcal{K} .
$$

As in [28] we also define the Banach spaces

$$
\mathcal{K}_{p}=\left\{g \in \mathcal{K}:\|g\|_{\mathcal{K}_{p}}=\|\|\left\|_{2}+\right\|\|\|_{p}<\infty\right\} \text { for } 1 \leq p \leq \infty .
$$

As before, we also define the Banach space $\tilde{\mathcal{K}}_{\infty}$ as the completion of

$$
\widetilde{\mathcal{K}}_{\infty}^{(0)}:=\left\{g \in C_{n}^{\infty}\left(\operatorname{Sym}^{+}(m)\right) \otimes C_{n}^{\infty}\left(\operatorname{Sym}^{+}(m)\right):\|g\|_{\infty}<\infty\right\}
$$

with respect to the norm $\|\cdot\|_{\infty}$.

Remark 2.2. The spaces $\mathscr{H}, \mathcal{H}_{p}, \tilde{\mathcal{H}}_{\infty}, \mathcal{K}, \mathcal{K}_{p}, \widetilde{\mathcal{K}}_{\infty}$, the supersymmetric Fourier transform $T$, etc. all depend on our choice of $n \geq \frac{m}{2}$ for a given $m$. This dependence on $n$ (and $m$ ) will be generally omitted.

\section{More supersymmetric identities}

In this section we derive new supersymmetric identities that are crucial for the extension of the results of [24] to the Bethe strip, going beyond the supersymmetric formalism used in [28] 
Using the first replica, we define the following Grassmann column vectors

$$
\overrightarrow{\bar{\Psi}}:=\left(\bar{\psi}_{1,1}, \bar{\psi}_{2,1}, \ldots, \bar{\psi}_{m, 1}\right)^{\top}, \quad \vec{\Psi}:=\left(\psi_{1,1}, \psi_{2,1}, \ldots, \psi_{m, 1}\right)^{\top}
$$

which correspond to the first and second column vector of $\boldsymbol{\Psi}$. Even though we only use the first replica (the second index is always one) for these vectors we do not add an index 1 to $\vec{\Psi}$ or $\vec{\Psi}$. We want to avoid having too many indices later, when we use a corresponding notation for an indexed family of supermatrices. Given $\boldsymbol{f}=\left(f_{1}, \ldots, f_{m}\right)^{\top} \in[\Lambda(\Psi)]^{m \times 1}$, a column vector of elements of the Grassmann algebra, we set

$$
\overrightarrow{\bar{\Psi}} \cdot \boldsymbol{f}:=\overrightarrow{\bar{\Psi}}^{\top} \boldsymbol{f}=\sum_{k=1}^{m} \bar{\psi}_{k, 1} f_{k} \quad \text { and } \quad \vec{\Psi} \cdot \boldsymbol{f}:=\vec{\Psi}^{\top} \boldsymbol{f}=\sum_{k=1}^{m} \psi_{k, 1} f_{k} .
$$

For an $m \times m$ matrix $\boldsymbol{F}=\left(F_{j, k}\right)_{j, k \in \mathcal{I}} \in[\Lambda(\boldsymbol{\Psi})]^{m \times m}$ the expressions $\boldsymbol{F} \overrightarrow{\bar{\Psi}}$ and $\boldsymbol{F} \vec{\Psi}$ will be understood as matrix multiplication.

Remark 3.1. Vectors of elements of the Grassmann algebra $\Lambda(\Psi)$ and vectors of functions will always be considered as column vectors in matrix products. In particular, the sets $\Lambda(\Psi)^{m}$ and $\left[S_{n}\left(\operatorname{Sym}^{+}(m)\right)\right]^{m}$ will be identified with $[\Lambda(\Psi)]^{m \times 1}$ and $\left[S_{n}\left(\operatorname{Sym}^{+}(m)\right)\right]^{m \times 1}$, respectively.

Given $\boldsymbol{a}=\left(a_{1}, \ldots, a_{n}\right) \in(\mathfrak{P}(\mathcal{I}))^{n}$ and $k \leq\left|a_{1}\right|$ we let $a_{1 k}$ denote the $k$-th smallest element of $a_{1}$. Similarly, $\bar{a}_{1 k}$ will denote the $k$-th smallest element of $\bar{a}_{1}$ for $\overline{\boldsymbol{a}}=\left(\bar{a}_{1}, \ldots, \bar{a}_{n}\right) \in(\mathfrak{P}(\mathcal{I}))^{n}$. If $(\overline{\boldsymbol{a}}, \boldsymbol{a}) \in \mathcal{P}_{1}^{n}$ and $k=1,2, \ldots,\left|\bar{a}_{1}\right|$, we have $\left(\overline{\boldsymbol{a}}-\llbracket \bar{a}_{1 k} \rrbracket, \boldsymbol{a}\right) \in \mathcal{P}^{n}$, and for $(\overline{\boldsymbol{a}}, \boldsymbol{a}) \in \mathcal{P}_{-1}^{n}$ and $k=1,2, \ldots,\left|a_{1}\right|$ we have $\left(\overline{\boldsymbol{a}}, \boldsymbol{a}-\llbracket a_{1 k} \rrbracket\right) \in \mathscr{P}^{n}$. Given $\boldsymbol{f}=\left(f_{1}, \ldots, f_{m}\right)^{\bar{\top}^{1}} \in\left[\mathcal{S}_{n}\left(\operatorname{Sym}^{+}(m)\right)\right]^{m}$, we have

$$
\begin{aligned}
\vec{\Psi} \cdot \boldsymbol{f}\left(\boldsymbol{\Phi}^{\odot 2}\right) & =\sum_{k=1}^{m} \bar{\psi}_{k, 1} f_{k}\left(\boldsymbol{\Phi}^{\odot 2}\right) \\
& =\sum_{k=1}^{m} \sum_{(\bar{b}, \boldsymbol{b}) \in \mathcal{P}^{n}} D_{\overline{\boldsymbol{b}}, \boldsymbol{b}} f_{k}\left(\boldsymbol{\varphi}^{\odot 2}\right) \operatorname{sgn}(\boldsymbol{b}) \bar{\psi}_{k, 1} \Psi_{\overline{\boldsymbol{b}}, \boldsymbol{b}} \\
& =\sum_{(\overline{\boldsymbol{a}}, \boldsymbol{a}) \in \mathcal{P}_{1}^{n}} \sum_{k=1}^{\left|\bar{a}_{1}\right|} D_{\overline{\boldsymbol{a}}-\llbracket \bar{a}_{1 k} \rrbracket, \boldsymbol{a}} f_{\bar{a}_{1 k}}\left(\varphi^{\odot 2}\right) \operatorname{sgn}(\boldsymbol{a})(-1)^{k-1} \Psi_{\overline{\boldsymbol{a}}, \boldsymbol{a}} .
\end{aligned}
$$

The change in the sum is done by the relations $\boldsymbol{a}=\boldsymbol{b}, \overline{\boldsymbol{a}}-\llbracket \bar{a}_{1 k} \rrbracket=\overline{\boldsymbol{b}}$. Note that for $k \in \bar{b}_{1}$ we have $\bar{\psi}_{k, 1} \Psi_{\overline{\boldsymbol{b}}, \boldsymbol{b}}=0$. Similarly, we obtain

$$
\vec{\Psi} \cdot \boldsymbol{f}\left(\boldsymbol{\Phi}^{\odot 2}\right)=\sum_{(\overline{\boldsymbol{a}}, \boldsymbol{a}) \in \mathcal{P}_{-}^{n}} \sum_{k=1}^{\left|a_{1}\right|} D_{\overline{\boldsymbol{a}}, \boldsymbol{a}-\llbracket a_{1 k} \rrbracket} f_{a_{1 k}}\left(\varphi^{\odot 2}\right) \operatorname{sgn}(\overline{\boldsymbol{a}})(-1)^{k-1+\left|\bar{a}_{1}\right|} \Psi_{\overline{\boldsymbol{a}}, \boldsymbol{a}}
$$

In order to obtain the super Fourier transform of these expression we will expand $e^{i \boldsymbol{\Phi} \cdot \boldsymbol{\Phi}^{\prime}}$ in the Grassmann variables. 
Lemma 3.2. We have

$$
e^{i \boldsymbol{\Phi} \cdot \boldsymbol{\Phi}^{\prime}}=e^{i \boldsymbol{\varphi} \cdot \boldsymbol{\varphi}^{\prime}} \sum_{\overline{\boldsymbol{a}}, \boldsymbol{a} \in \mathfrak{P}(\mathcal{L})^{n}}\left\{\left(\frac{i}{2}\right)^{|(\overline{\boldsymbol{a}}, \boldsymbol{a})|} \frac{\operatorname{sgn}(\boldsymbol{a})}{\operatorname{sgn}(\overline{\boldsymbol{a}})}(-1)^{|\boldsymbol{a}|} s(\overline{\boldsymbol{a}}, \boldsymbol{a}) \Psi_{\overline{\boldsymbol{a}}, \boldsymbol{a}}^{\prime} \Psi_{\boldsymbol{a}, \overline{\boldsymbol{a}}}\right\},
$$

where

$$
|\boldsymbol{a}|:=\sum_{\ell=1}^{n}\left|a_{\ell}\right| \quad \text { for } \boldsymbol{a} \in \mathfrak{P}(\mathcal{I})^{n}, \quad|(\overline{\boldsymbol{a}}, \boldsymbol{a})|:=|\overline{\boldsymbol{a}}|+|\boldsymbol{a}| \quad \text { for } \overline{\boldsymbol{a}}, \boldsymbol{a} \in \mathfrak{P}(\mathcal{I})^{n},
$$

and

$$
s(\overline{\boldsymbol{a}}, \boldsymbol{a}):=\prod_{1 \leq \ell<\ell^{\prime} \leq n}(-1)^{\left(\left|\bar{a}_{\ell}\right|+\left|a_{\ell}\right|\right) \cdot\left(\left|\bar{a}_{\ell^{\prime}}\right|+\left|a_{\ell^{\prime}}\right|\right)} .
$$

Proof. Recalling that for non-commutative products we always use the convention that the indices are ordered, increasing from left to right, we have

$$
\begin{aligned}
e^{i \boldsymbol{\Phi} \cdot \boldsymbol{\Phi}^{\prime}} & =e^{i \boldsymbol{\varphi} \cdot \boldsymbol{\varphi}^{\prime}} \prod_{k=1}^{m} \prod_{\ell=1}^{n}\left[\left(1+\frac{i}{2} \bar{\psi}_{k, \ell} \psi_{k, \ell}^{\prime}\right)\left(1+\frac{i}{2} \bar{\psi}_{k, \ell}^{\prime} \psi_{k, \ell}\right)\right] \\
& =e^{i \boldsymbol{\varphi} \cdot \boldsymbol{\varphi}^{\prime}} \sum_{\overline{\boldsymbol{a}}, \boldsymbol{a} \in \mathfrak{P}(\mathcal{I})^{n}}\left\{\left(\frac{i}{2}\right)^{|(\overline{\boldsymbol{a}}, \boldsymbol{a})|} \prod_{\ell=1}^{n}\left[\prod_{k \in \bar{a}_{\ell}} \bar{\psi}_{k, \ell}^{\prime} \psi_{k, \ell} \prod_{j \in a_{\ell}} \bar{\psi}_{j, \ell} \psi_{j, \ell}^{\prime}\right]\right\} \\
& =e^{i \boldsymbol{\varphi} \cdot \boldsymbol{\varphi}^{\prime}} \sum_{\overline{\boldsymbol{a}}, \boldsymbol{a} \in \mathfrak{P}(\mathcal{I})^{n}}\left\{\left(\frac{i}{2}\right)^{|(\overline{\boldsymbol{a}}, \boldsymbol{a})|} \frac{\operatorname{sgn}(\boldsymbol{a})}{\operatorname{sgn}(\overline{\boldsymbol{a}})} \prod_{\ell=1}^{n}\left[\prod_{k \in \bar{a}_{\ell}} \bar{\psi}_{k, \ell}^{\prime} \prod_{k \in \bar{a}_{\ell}} \psi_{k, \ell} \prod_{j \in a_{\ell}} \bar{\psi}_{j, \ell} \prod_{j \in a_{\ell}} \psi_{j, \ell}^{\prime}\right]\right\} \\
& =e^{i \boldsymbol{\varphi} \cdot \boldsymbol{\varphi}^{\prime}} \sum_{\overline{\boldsymbol{a}}, \boldsymbol{a} \in \mathfrak{P}(\mathcal{I})^{n}}\left\{\left(\frac{i}{2}\right)^{|(\overline{\boldsymbol{a}}, \boldsymbol{a})|} \frac{\operatorname{sgn}(\boldsymbol{a})}{\operatorname{sgn}(\overline{\boldsymbol{a}})}(-1)^{|\boldsymbol{a}|}\right. \\
& \left.\times \prod_{\ell=1}^{n}\left[\prod_{k \in \bar{a}_{\ell}} \bar{\psi}_{k, \ell}^{\prime} \prod_{j \in a_{\ell}} \psi_{j, \ell}^{\prime} \prod_{j \in a_{\ell}} \bar{\psi}_{j, \ell} \prod_{k \in \bar{a}_{\ell}} \psi_{k, \ell}\right]\right\} \\
& =e^{i \boldsymbol{\varphi} \cdot \boldsymbol{\varphi}^{\prime}} \sum_{\overline{\boldsymbol{a}}, \boldsymbol{a} \in \mathfrak{P}(\mathcal{I})^{n}}\left\{\left(\frac{i}{2}\right)^{|(\overline{\boldsymbol{a}}, \boldsymbol{a})|} \frac{\operatorname{sgn}(\boldsymbol{a})}{\operatorname{sgn}(\overline{\boldsymbol{a}})}(-1)^{|\boldsymbol{a}|} s(\overline{\boldsymbol{a}}, \boldsymbol{a}) \Psi_{\overline{\boldsymbol{a}}, \boldsymbol{a}}^{\prime} \Psi_{\boldsymbol{a}, \overline{\boldsymbol{a}}\} .}\right.
\end{aligned}
$$

Note that for $(\overline{\boldsymbol{a}}, \boldsymbol{a}) \in \mathcal{P}_{k}^{n}$ and $\ell \geq 2$ we have $\left|\bar{a}_{\ell}\right|=\left|a_{\ell}\right|$, therefore

$$
s(\overline{\boldsymbol{a}}, \boldsymbol{a})=1 \quad \text { for all }(\overline{\boldsymbol{a}}, \boldsymbol{a}) \in \mathcal{P}_{k}^{n} \text { and all } k \in[-m, m] \cap \mathbb{Z}
$$

Now if we combine (3.2) and (3.4), using $\left[\frac{i}{2}\right]^{|(\overline{\boldsymbol{a}}, \boldsymbol{a})|}=2 i \frac{(-1)^{|\boldsymbol{a}|}}{4^{|\overline{\boldsymbol{a}}|}}$ for $(\overline{\boldsymbol{a}}, \boldsymbol{a}) \in \mathcal{P}_{1}^{n}$ 
as well as (2.18), (2.23), and (2.21) for the integral over $D \Psi$, then we obtain

$$
\begin{gathered}
\int e^{i \boldsymbol{\Phi} \cdot \boldsymbol{\Phi}^{\prime}} \overrightarrow{\vec{\Psi}} \cdot \boldsymbol{f}\left(\boldsymbol{\Phi}^{\odot 2}\right) D \boldsymbol{\Phi} \\
=\sum_{(\overline{\boldsymbol{a}}, \boldsymbol{a}) \in \mathcal{P}_{1}^{n}}\left\{\frac{\operatorname{sgn}(\boldsymbol{a}) \operatorname{sgn}\left(\overline{\boldsymbol{a}}^{\boldsymbol{c}}\right) \operatorname{sgn}(\mathcal{I})}{\operatorname{sgn}(\overline{\boldsymbol{a}}) \operatorname{sgn}\left(\boldsymbol{a}, \overline{\boldsymbol{a}}, \boldsymbol{a}^{\boldsymbol{c}}, \overline{\boldsymbol{a}}^{\boldsymbol{c}}\right)}(-1)^{m n} \Psi_{\overline{\boldsymbol{a}}, \boldsymbol{a}}^{\prime}\right. \\
\left.\times 2 i \frac{2^{m n}}{4^{|\overline{\boldsymbol{a}}|}} \sum_{k=1}^{\left|a_{1}^{\mathrm{c}}\right|}(-1)^{k-1}\left(\mathcal{F} D_{\boldsymbol{a}} \boldsymbol{c}-\llbracket a_{1 k}^{\mathrm{c}} \rrbracket, \overline{\boldsymbol{a}}^{\boldsymbol{c}} f_{a_{1 k}^{\mathrm{c}}}\right)\left(\boldsymbol{\varphi}^{\prime \odot 2}\right)\right\} \\
=2 i \sum_{(\overline{\boldsymbol{a}}, \boldsymbol{a}) \in \mathcal{P}_{1}^{n}}\left\{\sum_{k=1}^{\left|a_{1}^{\mathrm{c}}\right|}(-1)^{a_{1 k}^{\mathrm{c}}-k+1} D_{\overline{\boldsymbol{a}}, \boldsymbol{a}+\llbracket a_{1 k}^{\mathrm{c}} \rrbracket} T f_{a_{1 k}^{\mathrm{c}}}\left(\boldsymbol{\varphi}^{\prime \odot 2}\right)\right\} \operatorname{sgn}(\boldsymbol{a}) \Psi_{\overline{\boldsymbol{a}}, \boldsymbol{a}}^{\prime} .
\end{gathered}
$$

Note that $a_{1 k}^{\mathrm{c}}=\left(a_{1}^{\mathrm{c}}\right)_{k}$ denotes the $k$-th smallest element of the set $a_{1}^{\mathrm{c}}=\mathcal{I} \backslash a_{1}$. To get the sign in the last equation we used

$$
\begin{gathered}
\frac{\operatorname{sgn}(\overline{\boldsymbol{a}}) \operatorname{sgn}\left(\overline{\boldsymbol{a}}^{\boldsymbol{c}}\right) \operatorname{sgn}(\mathcal{I})}{\operatorname{sgn}\left(\boldsymbol{a}, \overline{\boldsymbol{a}}, \boldsymbol{a}^{\boldsymbol{c}}, \overline{\boldsymbol{a}}^{\boldsymbol{c}}\right)}(-1)^{m n} \operatorname{sgn}\left(\boldsymbol{a}+\llbracket a_{1 k}^{\mathrm{c}} \rrbracket, \overline{\boldsymbol{a}}\right)(-1)^{k-1} \\
=\frac{\operatorname{sgn}\left(\boldsymbol{a}+\llbracket a_{1 k}^{\mathrm{c}} \rrbracket, \overline{\boldsymbol{a}}, \boldsymbol{a}^{\boldsymbol{c}}-\llbracket a_{1 k}^{\mathrm{c}} \rrbracket, \overline{\boldsymbol{a}}^{\boldsymbol{c}}\right)}{\operatorname{sgn}\left(\boldsymbol{a}, \overline{\boldsymbol{a}}, \boldsymbol{a}^{\boldsymbol{c}}, \overline{\boldsymbol{a}}^{\boldsymbol{c}}\right)}(-1)^{k-1},
\end{gathered}
$$

and the fact that for $(\boldsymbol{a}, \overline{\boldsymbol{a}}) \in \mathcal{P}_{-1}^{n}$ we have

$$
\begin{aligned}
\Psi_{\boldsymbol{a}, \overline{\boldsymbol{a}}} \Psi_{\boldsymbol{a}} \boldsymbol{c}, \overline{\boldsymbol{a}}^{c} & =(-1)^{k-1} \Psi_{\boldsymbol{a}, \overline{\boldsymbol{a}}} \bar{\psi}_{a_{1 k}^{\mathrm{c}}, 1} \Psi_{\boldsymbol{a}}{ }^{c}-\llbracket a_{1 k}^{\mathrm{c}} \rrbracket, \overline{\boldsymbol{a}}^{c}=(-1)^{k} \bar{\psi}_{a_{1 k}^{\mathrm{c}}, 1} \Psi_{\boldsymbol{a}, \overline{\boldsymbol{a}}} \Psi_{\boldsymbol{a}}{ }^{c}-\llbracket a_{1 k}^{\mathrm{c}} \rrbracket, \overline{\boldsymbol{a}}^{c} \\
& =(-1)^{k}(-1)^{a_{1 k}^{\mathrm{c}}-k} \Psi_{\boldsymbol{a}+\llbracket a_{1 k}^{\mathrm{c}} \rrbracket, \overline{\boldsymbol{a}}} \Psi_{\boldsymbol{a}^{c}-\llbracket a_{1 k}^{\mathrm{c}} \rrbracket, \overline{\boldsymbol{a}} \boldsymbol{c},}
\end{aligned}
$$

implying

$$
\frac{\operatorname{sgn}\left(\boldsymbol{a}+\llbracket a_{1 k}^{\mathrm{c}} \rrbracket, \overline{\boldsymbol{a}}, \boldsymbol{a}^{\boldsymbol{c}}-\llbracket a_{1 k}^{\mathrm{c}} \rrbracket, \overline{\boldsymbol{a}}^{\boldsymbol{c}}\right)}{\operatorname{sgn}\left(\boldsymbol{a}, \overline{\boldsymbol{a}}, \boldsymbol{a}^{\boldsymbol{c}}, \overline{\boldsymbol{a}}^{\boldsymbol{c}}\right)}=(-1)^{a_{1 k}^{\mathrm{c}}} .
$$

Since for $(\overline{\boldsymbol{a}}, \boldsymbol{a}) \in \mathcal{P}_{-1}^{n}$ we have

$$
\left(\frac{i}{2}\right)^{|(\bar{a}, \boldsymbol{a})|}=-2 i \frac{(-1)^{|\boldsymbol{a}|}}{4^{|\boldsymbol{a}|}}
$$

and

$$
\frac{\operatorname{sgn}\left(\boldsymbol{a}, \overline{\boldsymbol{a}}+\llbracket \bar{a}_{1 k}^{\mathrm{c}} \rrbracket, \boldsymbol{a}^{\boldsymbol{c}}, \overline{\boldsymbol{a}}^{\boldsymbol{c}}-\llbracket \bar{a}_{1 k}^{\mathrm{c}} \rrbracket\right)}{\operatorname{sgn}\left(\boldsymbol{a}, \overline{\boldsymbol{a}}, \boldsymbol{a}^{\boldsymbol{c}}, \overline{\boldsymbol{a}}^{\boldsymbol{c}}\right)}=(-1)^{m+a_{1 k}^{\mathrm{c}}},
$$


similar calculations lead to

$$
\begin{aligned}
& \int e^{i \boldsymbol{\Phi} \cdot \boldsymbol{\Phi}^{\prime}} \vec{\Psi} \cdot \boldsymbol{f}\left(\boldsymbol{\Phi}^{\odot 2}\right) D \boldsymbol{\Phi} \\
& =-2 i \sum_{(\overline{\boldsymbol{a}}, \boldsymbol{a}) \in \mathcal{P}_{-1}^{n}}\left\{\sum_{k=1}^{\left|\bar{a}_{1}^{\mathrm{c}}\right|}(-1)^{m+\bar{a}_{1 k}^{\mathrm{c}}+k-1+\left|a_{1}^{\mathrm{c}}\right|} D_{\overline{\boldsymbol{a}}+\llbracket \bar{a}_{1 k}^{\mathrm{c}} \|, \boldsymbol{a}}\right. \\
& \left.\times T f_{\bar{a}_{1 k}^{\mathrm{c}}}\left(\varphi^{\prime \odot 2}\right)\right\} \operatorname{sgn}(\overline{\boldsymbol{a}}) \Psi_{\overline{\boldsymbol{a}}, \boldsymbol{a}}^{\prime} \\
& =-2 i \sum_{(\overline{\boldsymbol{a}}, \boldsymbol{a}) \in \mathcal{P}_{-1}^{n}}\left\{\sum_{k=1}^{\left|\bar{a}_{1}^{\mathrm{c}}\right|}(-1)^{\bar{a}_{1 k}^{\mathrm{c}}-k+\left|\bar{a}_{1}\right|} D_{\overline{\boldsymbol{a}}+\llbracket \bar{a}_{1 k}^{\mathrm{c}} \rrbracket, \boldsymbol{a}} T f_{\bar{a}_{1 k}^{\mathrm{c}}}\left(\varphi^{\prime \odot 2}\right)\right\} \operatorname{sgn}(\overline{\boldsymbol{a}}) \Psi_{\overline{\boldsymbol{a}}, \boldsymbol{a}}^{\prime} .
\end{aligned}
$$

We are now ready to derive the main identities we will need to prove Theorem 1.2. The integrals (3.5) and (3.6) can be expressed using the matrix operator $\mathbb{T}$ defined by

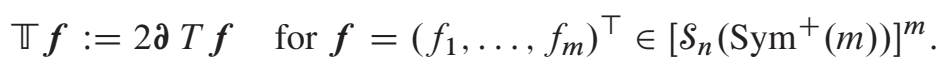

Combining (2.26), (2.15), (2.25) and (2.24) we see that $\mathbb{T}$ is an involution,

$$
\mathbb{T}^{2} \boldsymbol{f}=4 \boldsymbol{\partial} T \boldsymbol{\partial} T \boldsymbol{f}=4(-2)^{m n-2} \mathcal{F} \delta^{n-1} \boldsymbol{\partial} \boldsymbol{\partial} T \boldsymbol{f}=(-2)^{m n} \mathscr{F} \boldsymbol{\delta}^{n} T \boldsymbol{f}=T^{2} \boldsymbol{f}=\boldsymbol{f} .
$$

The following result is crucial for this article. It is the key observation that allows the extension of the results in [24] to the Bethe strip.

Theorem 3.3. Let $\boldsymbol{f}=\left(f_{1}, f_{2} \ldots, f_{m}\right)^{\top} \in\left[S_{n}\left(\operatorname{Sym}^{+}(m)\right)\right]^{m}$. Then

$$
\begin{aligned}
\vec{\Psi}^{\prime} \cdot \mathbb{f}\left(\boldsymbol{\Phi}^{\prime \odot 2}\right) & =i \int e^{i \boldsymbol{\Phi} \cdot \boldsymbol{\Phi}^{\prime}} \overrightarrow{\bar{\Psi}} \cdot \boldsymbol{f}\left(\boldsymbol{\Phi}^{\odot 2}\right) D \boldsymbol{\Phi} \\
& =-i \int e^{-i \boldsymbol{\Phi} \cdot \boldsymbol{\Phi}^{\prime}} \overrightarrow{\bar{\Psi}} \cdot \boldsymbol{f}\left(\boldsymbol{\Phi}^{\odot 2}\right) D \boldsymbol{\Phi},
\end{aligned}
$$

and

$$
\begin{aligned}
\vec{\Psi}^{\prime} \cdot \mathbb{f}\left(\boldsymbol{\Phi}^{\prime \odot 2}\right) & =i \int e^{i \boldsymbol{\Phi} \cdot \boldsymbol{\Phi}^{\prime}} \vec{\Psi} \cdot \boldsymbol{f}\left(\boldsymbol{\Phi}^{\odot 2}\right) D \boldsymbol{\Phi} \\
& =-i \int e^{-i \boldsymbol{\Phi} \cdot \boldsymbol{\Phi}^{\prime}} \vec{\Psi} \cdot \boldsymbol{f}\left(\boldsymbol{\Phi}^{\odot 2}\right) D \boldsymbol{\Phi} .
\end{aligned}
$$

Proof. The second equalities in (3.8) and (3.9) follow from a simple change of variables. Using (3.2) we get

$$
\begin{array}{r}
\overrightarrow{\bar{\Psi}}^{\prime} \cdot \partial T \boldsymbol{f}\left(\boldsymbol{\Phi}^{\prime \odot 2}\right)=\sum_{(\overline{\boldsymbol{a}}, \boldsymbol{a}) \in \mathcal{P}_{1}^{n}}\left\{\sum_{k=1}^{\left|\bar{a}_{1}\right|} \sum_{k^{\prime}=1}^{m}(-1)^{k-1} D_{\overline{\boldsymbol{a}}-\llbracket \bar{a}_{1 k} \rrbracket, \boldsymbol{a}} D_{\left\{a_{1 k}\right\},\left\{k^{\prime}\right\}}\right. \\
\left.\times T f_{k^{\prime}}\left(\boldsymbol{\varphi}^{\prime \odot 2}\right)\right\} \operatorname{sgn}(\boldsymbol{a}) \Psi_{\overline{\boldsymbol{a}}, \boldsymbol{a}}^{\prime} .
\end{array}
$$

First consider the case $k^{\prime}=a_{1 j}^{\mathrm{c}}$. There are $a_{1 j}^{\mathrm{c}}-1$ numbers smaller than $a_{1 j}^{\mathrm{c}}$ in $\mathcal{I}$, $j-1$ of them are in the set $a_{1}^{\mathrm{c}}$ and hence $a_{1 j}^{\mathrm{c}}-j$ of them are in the set $a_{1}$. Therefore, 
$a_{1 j}^{\mathrm{c}}$ is the $\left(a_{1 j}^{\mathrm{c}}-j+1\right)$-th smallest element of the set $a_{1} \cup\left\{a_{1 j}^{\mathrm{c}}\right\}$. A column expansion of $D_{\bar{a}_{1}, a_{1} \cup\left\{a_{1 j}^{\mathrm{c}}\right\}}$, the determinant of $\boldsymbol{\partial}_{\bar{a}_{1}, a_{1} \cup\left\{a_{1 j}^{\mathrm{c}}\right\}}$, leads to

$$
D_{\bar{a}_{1}, a_{1} \cup\left\{a_{1 j}^{\mathrm{c}}\right\}}=\sum_{k=1}^{\left|\bar{a}_{1}\right|}(-1)^{k+a_{1 j}^{\mathrm{c}}-j+1} D_{\bar{a}_{1} \backslash\left\{\bar{a}_{1 k}\right\}, a_{1}} D_{\left\{\bar{a}_{1 k}\right\},\left\{a_{1 j}^{\mathrm{c}}\right\}},
$$

implying

$$
\sum_{k=1}^{\left|\bar{a}_{1}\right|}(-1)^{k-1} D_{\overline{\boldsymbol{a}}-\llbracket \bar{a}_{1 k} \rrbracket, \boldsymbol{a}} D_{\left\{a_{1 k}\right\},\left\{a_{1 j}^{\mathrm{c}}\right\}}=(-1)^{a_{1 j}^{\mathrm{c}}-j} D_{\overline{\boldsymbol{a}}, \boldsymbol{a}+\llbracket a_{1 j}^{\mathrm{c}} \rrbracket} .
$$

Similarly, for $k^{\prime} \in a_{1}$ we can also interpret the sum over $k$ as the expansion of a determinant. However, in this case the corresponding matrix has two identical rows, therefore

$$
\sum_{k=1}^{\left|\bar{a}_{1}\right|}(-1)^{k-1} D_{\overline{\boldsymbol{a}}-\llbracket \bar{a}_{1 k} \rrbracket, \boldsymbol{a}} D_{\left\{a_{1 k}\right\},\left\{k^{\prime}\right\}} T f_{k^{\prime}}\left(\varphi^{\prime \odot 2}\right)=0 \quad \text { for } k^{\prime} \in a_{1} .
$$

Now equations (3.10), (3.11) and (3.12) lead to

$$
\begin{array}{r}
\overrightarrow{\bar{\Psi}}^{\prime} \cdot \partial T \boldsymbol{f}\left(\boldsymbol{\Phi}^{\prime \odot 2}\right)=\sum_{(\overline{\boldsymbol{a}}, \boldsymbol{a}) \in \mathcal{P}_{1}^{n}}\left\{\sum_{j=1}^{\left|a_{1}^{\mathrm{c}}\right|}(-1)^{a_{1 j}^{\mathrm{c}}-j} D_{\overline{\boldsymbol{a}}, \boldsymbol{a}+\llbracket a_{1 j}^{\mathrm{c}} \|}\right. \\
\left.\times T f_{a_{1 j}^{\mathrm{c}}}\left(\boldsymbol{\varphi}^{\prime \odot 2}\right)\right\} \operatorname{sgn}(\boldsymbol{a}) \Psi_{\overline{\boldsymbol{a}}, \boldsymbol{a}}^{\prime},
\end{array}
$$

which combined with (3.5) proves (3.8).

For the second equation one starts from (3.3); similar calculations lead to

$$
\begin{aligned}
\vec{\Psi}^{\prime} \cdot \partial T \boldsymbol{f}\left(\boldsymbol{\Phi}^{\prime \odot 2}\right)= & \sum_{(\overline{\boldsymbol{a}}, \boldsymbol{a}) \in \mathcal{P}_{-1}^{n}}\left\{\sum_{j=1}^{\left|a_{1}^{\mathrm{c}}\right|}(-1)^{a_{1 j}^{\mathrm{c}}-j+\left|\bar{a}_{1}\right|} D_{\overline{\boldsymbol{a}}+\llbracket \bar{a}_{1 j}^{\mathrm{c}} \rrbracket, \boldsymbol{a}}\right. \\
& \left.\times T f_{\bar{a}_{1 j}^{\mathrm{c}}}\left(\boldsymbol{\varphi}^{\prime \odot 2}\right)\right\} \operatorname{sgn}(\boldsymbol{a}) \Psi_{\overline{\boldsymbol{a}}, \boldsymbol{a}}^{\prime} .
\end{aligned}
$$

Combining this with (3.6) yields (3.9).

Next we introduce a Hilbert space on which $\mathbb{T}$ is a unitary operator. In view of (3.2), we define differential operators on $\left[C_{n}^{\infty}\left(\operatorname{Sym}^{+}(m)\right)\right]^{m}$ by

$$
\mathbb{D}_{\overline{\boldsymbol{a}}, \boldsymbol{a}} \boldsymbol{f}:=\operatorname{sgn}(\boldsymbol{a}) \sum_{k=1}^{\left|\bar{a}_{1}\right|}(-1)^{k-1} D_{\overline{\boldsymbol{a}}-\llbracket \bar{a}_{1 k} \rrbracket, \boldsymbol{a}} f_{\bar{a}_{1 k}} \quad \text { for }(\overline{\boldsymbol{a}}, \boldsymbol{a}) \in \mathcal{P}_{1}^{n},
$$

where $\boldsymbol{f}=\left(f_{1}, \ldots, f_{m}\right)^{\top} \in\left[C_{n}^{\infty}\left(\operatorname{Sym}^{+}(m)\right)\right]^{m}$. $\mathbb{D}_{\overline{\boldsymbol{a}}, \boldsymbol{a}}$ may be considered as a rowvector of differential operators. Equations (3.2) and (3.3) can be written as

$$
\overrightarrow{\bar{\Psi}} \cdot \boldsymbol{f}\left(\Phi^{\odot 2}\right)=\sum_{(\bar{a}, \boldsymbol{a}) \in \mathcal{P}_{1}^{n}} \mathbb{D}_{\bar{a}, \boldsymbol{a}} \boldsymbol{f}\left(\varphi^{\odot 2}\right) \Psi_{\overline{\boldsymbol{a}}, \boldsymbol{a}}
$$


and

$$
\vec{\Psi} \cdot \boldsymbol{f}\left(\boldsymbol{\Phi}^{\odot 2}\right)=\sum_{(\bar{a}, \boldsymbol{a}) \in \mathcal{P}_{-1}^{n}}(-1)^{\left|\bar{a}_{1}\right|} \mathbb{D}_{\boldsymbol{a}, \overline{\boldsymbol{a}}} \boldsymbol{f}\left(\varphi^{\odot 2}\right) \Psi_{\overline{\boldsymbol{a}}, \boldsymbol{a}} .
$$

Combining Theorem 3.3 with (3.5) and (3.15), we obtain

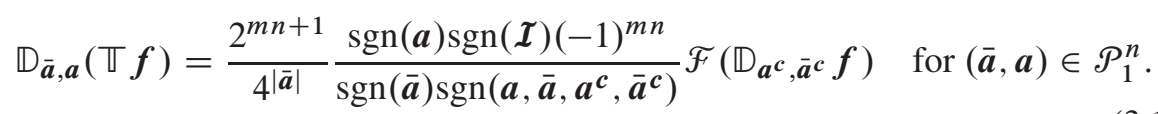

This leads us to define the norm

$$
\|\boldsymbol{f}\|_{\mathbb{Q}}^{2}:=\sum_{(\overline{\boldsymbol{a}}, \boldsymbol{a}) \in \mathcal{P}_{1}^{n}}\left\|2^{|\boldsymbol{a}|} \mathbb{D}_{\overline{\boldsymbol{a}}, \boldsymbol{a}} \boldsymbol{f}\left(\boldsymbol{\varphi}^{\odot 2}\right)\right\|_{L^{2}\left(\mathbb{R}^{m \times 2 n} ; d^{2 m n} \boldsymbol{\varphi}\right)}^{2},
$$

and let 때 be the Hilbert space completion of $\left[S_{n}\left(\operatorname{Sym}^{+}(m)\right)\right]^{m}$ with respect to the norm $\|\cdot\|_{\mathbb{Q}}$. By (3.17), 『 extends to a unitary operator on $\mathbb{H}$. Moreover, the expressions $\vec{\Psi} \cdot f$ and $\vec{\Psi} \cdot f$ can be extended to $f \in \mathbb{T}$ and the equations (3.8) and (3.9) remain valid.

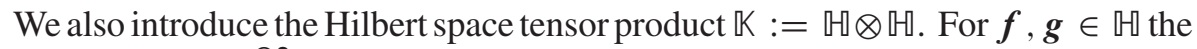
tensor product $f\left(\varphi_{+}^{\odot 2}\right) \otimes g\left(\varphi_{-}^{\odot 2}\right)$ can be identified with the matrix valued function given by the matrix product $f\left(\varphi_{+}^{\odot 2}\right)\left[g\left(\varphi_{-}^{\odot 2}\right)\right]^{\top}$. With this identification, the norm for an $m \times m$ matrix valued function $\boldsymbol{F}\left(\boldsymbol{\varphi}_{+}^{\odot 2}, \boldsymbol{\varphi}_{-}^{\odot 2}\right) \in \mathbb{K}$ is given by

$$
\|\boldsymbol{F}\|_{\mathbb{K}}^{2}
$$

where $\mathbb{D}_{\overline{\boldsymbol{a}}, \boldsymbol{a}}^{( \pm)}$denotes the operator $\mathbb{D}_{\overline{\boldsymbol{a}}, \boldsymbol{a}}$ acting with respect to $\varphi_{ \pm}^{\odot 2}$. To obtain (3.19), note that $\mathbb{D}_{\overline{\boldsymbol{a}}, \boldsymbol{a}}^{( \pm)}$are $1 \times m$ row-vectors of differential operators and hence $\mathbb{D}_{\overline{\boldsymbol{a}}, \boldsymbol{a}}^{(+)} \boldsymbol{f}\left(\boldsymbol{\varphi}_{+}^{\odot 2}\right)=$ $\left[\mathbb{D}_{\overline{\boldsymbol{a}}, \boldsymbol{a}}^{(+)} \boldsymbol{f}\left(\varphi_{+}^{\odot 2}\right)\right]^{\top}$, since it is a $1 \times 1$ matrix, which leads to

$$
\left.\left[\mathbb{D}_{\overline{\boldsymbol{a}}, \boldsymbol{a}}^{(+)} \boldsymbol{f}\left(\varphi_{+}^{\odot 2}\right)\right]\left[\mathbb{D}_{\overline{\boldsymbol{b}}, \boldsymbol{b}}^{(-)} \boldsymbol{g}\left(\varphi_{-}^{\odot 2}\right)\right]=\mathbb{D}_{\overline{\boldsymbol{b}}, \boldsymbol{b}}^{(-)}\left[\mathbb{D}_{\overline{\boldsymbol{a}}, \boldsymbol{a}}^{(+)} \boldsymbol{f}\left(\varphi_{+}^{\odot 2}\right) \boldsymbol{g}^{\top}\left(\varphi_{-}^{\odot 2}\right)\right)\right]^{\top} .
$$

Together with (3.15) and (3.16) this calculation also implies

$$
\overrightarrow{\bar{\Psi}}_{+} \cdot \boldsymbol{F}\left(\boldsymbol{\Phi}_{+}^{\odot 2}, \boldsymbol{\Phi}_{-}^{\odot 2}\right) \overrightarrow{\bar{\Psi}}_{-}=\sum_{\substack{(\overline{\boldsymbol{a}}, \boldsymbol{a}) \in \mathcal{P}_{1}^{n} \\(\overline{\boldsymbol{b}}, \boldsymbol{b}) \in \mathcal{P}_{1}^{n}}} \mathbb{D}_{\overline{\boldsymbol{b}}, \boldsymbol{b}}^{(-)}\left[\mathbb{D}_{\overline{\boldsymbol{a}}, \boldsymbol{a}}^{(+)} \boldsymbol{F}\left(\varphi_{+}^{\odot 2}, \boldsymbol{\varphi}_{-}^{\odot 2}\right)\right]^{\top} \Psi_{+, \overline{\boldsymbol{a}}, \boldsymbol{a}} \Psi_{-, \overline{\boldsymbol{b}}, \boldsymbol{b}}
$$

and

$$
\begin{aligned}
& \vec{\Psi}_{+} \cdot \boldsymbol{F}\left(\boldsymbol{\Phi}_{+}^{\odot 2}, \boldsymbol{\Phi}_{-}^{\odot 2}\right) \vec{\Psi}_{-} \\
& \quad=\sum_{\substack{(\overline{\boldsymbol{a}}, \boldsymbol{a}) \in \mathcal{P}_{-1}^{n} \\
(\overline{\boldsymbol{b}}, \boldsymbol{b}) \in \mathcal{P}_{-1}^{n}}}(-1)^{\left|\bar{a}_{1}\right|+\left|\bar{b}_{1}\right|} \mathbb{D}_{\boldsymbol{b}, \overline{\boldsymbol{b}}}^{(-)}\left[\mathbb{D}_{\boldsymbol{a}, \overline{\boldsymbol{a}}}^{(+)} \boldsymbol{F}\left(\varphi_{+}^{\odot 2}, \varphi_{-}^{\odot 2}\right)\right]^{\top} \Psi_{+, \overline{\boldsymbol{a}}, \boldsymbol{a}} \Psi_{-, \bar{b}, \boldsymbol{b}},
\end{aligned}
$$


where $\Psi_{+, \overline{\boldsymbol{a}}, \boldsymbol{a}}$ and $\Psi_{-, \overline{\boldsymbol{b}}, \boldsymbol{b}}$ are defined analogously to $\Psi_{\overline{\boldsymbol{a}}, \boldsymbol{a}}$ using the Grassmann entries of $\boldsymbol{\Phi}_{+}$and $\boldsymbol{\Phi}_{-}$, respectively. An important operator on $\mathbb{K}$ is the tensor operator $\widehat{\mathbb{T}}:=\mathbb{\mathbb { V }} \otimes \mathbb{T}$. Theorem 3.3 implies the following.

Corollary 3.4. Let $\boldsymbol{F}\left(\boldsymbol{\Phi}_{+}^{\odot 2}, \boldsymbol{\Phi}_{-}^{\odot 2}\right) \in \mathbb{K}$. Then

$$
\begin{aligned}
\overrightarrow{\bar{\Psi}}_{+}^{\prime} & \cdot \hat{\mathbb{T}} \boldsymbol{F}\left(\boldsymbol{\Phi}_{+}^{\prime \odot 2}, \boldsymbol{\Phi}_{-}^{\prime \odot 2}\right) \overrightarrow{\bar{\Psi}}_{-}^{\prime} \\
& =\int e^{ \pm i\left(\boldsymbol{\Phi}_{+} \cdot \boldsymbol{\Phi}_{+}^{\prime}-\boldsymbol{\Phi}_{-} \cdot \boldsymbol{\Phi}_{-}^{\prime}\right)} \overrightarrow{\bar{\Psi}}_{+} \cdot \boldsymbol{F}\left(\boldsymbol{\Phi}_{+}^{\odot 2}, \boldsymbol{\Phi}_{-}^{\odot 2}\right) \overrightarrow{\bar{\Psi}}_{-} D \boldsymbol{\Phi}_{+} D \boldsymbol{\Phi}_{-}
\end{aligned}
$$

and

$$
\begin{aligned}
\vec{\Psi}_{+}^{\prime} & . \widehat{\mathbb{T}} \boldsymbol{F}\left(\boldsymbol{\Phi}_{+}^{\prime \odot 2}, \boldsymbol{\Phi}_{-}^{\prime \odot 2}\right) \vec{\Psi}_{-}^{\prime} \\
& =\int e^{ \pm i\left(\boldsymbol{\Phi}_{+} \cdot \boldsymbol{\Phi}_{+}^{\prime}-\boldsymbol{\Phi}_{-} \cdot \boldsymbol{\Phi}_{-}^{\prime}\right)} \vec{\Psi}_{+} \cdot \boldsymbol{F}\left(\boldsymbol{\Phi}_{+}^{\odot 2}, \boldsymbol{\Phi}_{-}^{\odot 2}\right) \vec{\Psi}_{-} D \boldsymbol{\Phi}_{+} D \boldsymbol{\Phi}_{-} .
\end{aligned}
$$
norms

We recall that $\mathscr{H}^{m}=\bigoplus_{k=1}^{m} \mathscr{H}$ and $\mathcal{K}^{m \times m} \cong \mathcal{K}^{m^{2}}$ are Hilbert spaces with the

$$
\begin{gathered}
\|\boldsymbol{f}\|_{\mathscr{H}^{m}}^{2}=\sum_{k=1}^{m}\left\|f_{k}\right\|_{2}^{2} \quad \text { for } \boldsymbol{f}=\left(f_{1}, \ldots, f_{m}\right) \in \mathscr{H}^{m}, \\
\|\boldsymbol{F}\|_{\mathcal{K}^{m \times m}}^{2}=\sum_{j, k=1}^{m}\left\|F_{j k}\right\|_{2}^{2} \quad \text { for } \boldsymbol{F}=\left(F_{j k}\right) \in \mathcal{K}^{m \times m} .
\end{gathered}
$$

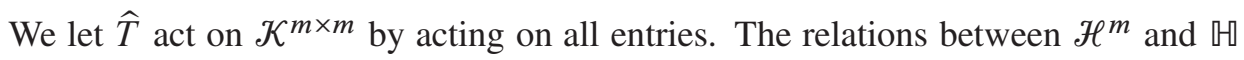
and between $\mathcal{K}^{m \times m}$ and $\mathbb{K}$ will play a crucial role.

Proposition 3.5. (i) $\mathscr{H}^{m}$ is a subset of $\mathbb{H}$ and the canonical injection $\mathscr{H}^{m} \mapsto \mathbb{\square}$ is continuous with respect to the norms of $\mathscr{H}^{m}$ and $\mathbb{H}$.

(ii) $\mathcal{K}^{m \times m}$ is a subset of $\mathbb{K}$ and the canonical injection $\mathcal{K}^{m \times m} \rightarrow \mathbb{K}$ is continuous with respect to the norms of $\mathcal{K}^{m \times m}$ and $\mathbb{K}$.

(iii) The matrix differential operator d acting on $\left[S_{n}\left(\mathrm{Sym}^{+}(m)\right)\right]^{m}$ extends to a continuous operator from $\mathscr{H}^{m}$ to $\mathbb{H}$, and we have

$$
2 \partial \boldsymbol{f}=\mathbb{T} T \boldsymbol{f} \text { for all } \boldsymbol{f} \in \mathscr{H}^{m} \text {. }
$$

(iv) The operators $\mathbf{\partial} \otimes \mathbf{1}, \mathbf{1} \otimes \mathbf{\partial}$ and $\mathbf{\partial} \otimes \mathbf{\partial}$ are continuous from $\mathcal{K}^{m \times m}$ to $\mathbb{K}$. They are given by

$$
\begin{aligned}
& (\boldsymbol{\partial} \otimes \mathbf{1}) \boldsymbol{F}\left(\boldsymbol{\Phi}_{+}^{\odot 2}, \boldsymbol{\Phi}_{-}^{\odot 2}\right)=\partial_{+} \boldsymbol{F}\left(\boldsymbol{\Phi}_{+}^{\odot 2}, \boldsymbol{\Phi}_{-}^{\odot 2}\right), \\
& (\mathbf{1} \otimes \partial) \boldsymbol{F}\left(\boldsymbol{\Phi}_{+}^{\odot 2}, \boldsymbol{\Phi}_{-}^{\odot 2}\right)=\left[\partial_{-} \boldsymbol{F}^{\top}\left(\boldsymbol{\Phi}_{+}^{\odot 2}, \boldsymbol{\Phi}_{-}^{\odot 2}\right)\right]^{\top}, \\
& (\boldsymbol{\partial} \otimes \partial) \boldsymbol{F}\left(\boldsymbol{\Phi}_{+}^{\odot 2}, \boldsymbol{\Phi}_{-}^{\odot 2}\right)=\left\{\partial_{-}\left[\partial_{+} \boldsymbol{F}\left(\boldsymbol{\Phi}_{+}^{\odot 2}, \boldsymbol{\Phi}_{-}^{\odot 2}\right)\right]^{\top}\right\}^{\top},
\end{aligned}
$$


where $\partial_{ \pm}$is the matrix of differential operators $\boldsymbol{\partial}$ with respect to $\boldsymbol{\Phi}_{ \pm}$. (The products are matrix products.)

(v) We have

$$
\widehat{\mathbb{U}} \boldsymbol{F}=4\left[\boldsymbol{\partial}_{-}\left(\boldsymbol{\partial}_{+} \hat{T} \boldsymbol{F}\right)^{\top}\right]^{\top} \text { for all } \boldsymbol{F} \in \mathbb{K} .
$$

(v) Given $g\left(\varphi^{\odot 2}\right) \in \tilde{\mathscr{H}}_{\infty}^{(0)}$, the multiplication operator $M(g)$, defined by

$$
M(g) \boldsymbol{f}\left(\boldsymbol{\varphi}^{\odot 2}\right)=g\left(\boldsymbol{\varphi}^{\odot 2}\right) \boldsymbol{f}\left(\boldsymbol{\varphi}^{\odot 2}\right) \text { for } \boldsymbol{f} \in\left[\mathcal{S}_{n}\left(\operatorname{Sym}^{+}(m)\right)\right]^{m},
$$

extends to a bounded operator on $\mathbb{W}$. The map $g \in \mathscr{H}_{\infty}^{(0)} \mapsto M(g) \in B(\mathbb{U})$ is continuous, and hence extends to $\mathscr{H}_{\infty}$. Moreover, for $(\overline{\boldsymbol{a}}, \boldsymbol{a}) \in \mathcal{P}_{1}^{n}$ we have

$$
\mathbb{D}_{\overline{\boldsymbol{a}}, \boldsymbol{a}}(g \boldsymbol{f})=\sum_{\substack{(\overline{\boldsymbol{b}}, \boldsymbol{b}) \in \mathcal{P}^{n},\left(\overline{\boldsymbol{b}}^{\prime}, \boldsymbol{b}^{\prime}\right) \in \mathcal{P}_{1}^{n} \\(\overline{\boldsymbol{b}}, \boldsymbol{b})+\left(\overline{\boldsymbol{b}}^{\prime}, \boldsymbol{b}^{\prime}\right)=(\overline{\boldsymbol{a}}, \boldsymbol{a})}} \operatorname{sgn}(\boldsymbol{b}) \operatorname{sgn}\left(\overline{\boldsymbol{b}}, \boldsymbol{b}, \overline{\boldsymbol{b}}^{\prime}, \boldsymbol{b}^{\prime}\right) D_{\overline{\boldsymbol{b}}, \boldsymbol{b}} g \mathbb{D}_{\overline{\boldsymbol{b}}^{\prime}, \boldsymbol{b}^{\prime}} \boldsymbol{f} .
$$

(vi) Given $G\left(\varphi_{+}^{\odot 2}, \varphi_{-}^{\odot 2}\right) \in \mathcal{K}_{\infty}^{(0)}$, the multiplication operator $M(G)$, defined by

$$
M(G) \boldsymbol{F}\left(\varphi_{+}^{\odot 2}, \varphi_{-}^{\odot 2}\right)=G\left(\varphi_{+}^{\odot 2}, \varphi_{-}\right) \boldsymbol{F}\left(\varphi_{+}^{\odot 2}, \varphi_{-}^{\odot 2}\right),
$$

for $\boldsymbol{F} \in\left[S_{n}\left(\operatorname{Sym}^{+}(m)\right)\right]^{m} \otimes\left[S_{n}\left(\operatorname{Sym}^{+}(m)\right)\right]^{m}$, extends to a bounded operator on $\mathbb{K}$. The map $G \in \mathcal{K}_{\infty}^{(0)} \mapsto M(G) \in B(\mathbb{K})$ is continuous, and hence extends to $\mathcal{K}_{\infty}$.

Proof. (i) is a simple consequence of the definitions of the norm. (ii) follows from (i) since $\mathscr{H}^{m} \otimes \mathscr{H}^{m} \cong \mathcal{K}^{m \times m}$.

To get (iii) note that for $\boldsymbol{f} \in\left[S_{n}\left(\operatorname{Sym}^{+}(m)\right)\right]^{m}$ (3.22) follows from (2.24) and (3.7). Since $T$ is unitary on $\mathscr{H}^{m}, \mathscr{H}^{m}$ is continuously embedded in $\mathbb{U}$ by (i), and

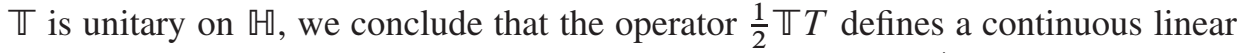
map from $\mathscr{H}^{m}$ to $\mathbb{W}$ which extends the map $\boldsymbol{f} \in\left[S_{n}\left(\operatorname{Sym}^{+}(m)\right)\right]^{m} \mapsto \partial \boldsymbol{f} \in$ $\left[S_{n}\left(\operatorname{Sym}^{+}(m)\right)\right]^{m}$.

The continuity in (iv) follows from (iii). For the second and third equation note that $\boldsymbol{\partial}=\boldsymbol{\partial}^{\top}$ and hence

$$
\begin{aligned}
(1 \otimes \partial)\left(f\left(\varphi_{+}^{\odot 2}\right) \otimes g\left(\varphi_{-}^{\odot 2}\right)\right) & =f\left(\varphi_{+}^{\odot 2}\right)\left[\partial_{-} g\left(\varphi_{-}^{\odot 2}\right)\right]^{\top} \\
& =\left[\partial_{-} g\left(\varphi_{-}^{\odot 2}\right) f^{\top}\left(\varphi_{+}^{\odot 2}\right)\right]^{\top} \\
& =\left[\partial_{-}\left[f\left(\varphi_{+}^{\odot 2}\right) g^{\top}\left(\varphi_{-}^{\odot 2}\right)\right]^{\top}\right]^{\top} .
\end{aligned}
$$

(v) follows from (iv) and (3.7). To prove (vi) note that $\overrightarrow{\bar{\Psi}} \cdot M(g) \boldsymbol{f}\left(\boldsymbol{\Phi}^{\odot 2}\right)=$ $g\left(\boldsymbol{\Phi}^{\odot 2}\right) \overrightarrow{\bar{\Psi}} \cdot \boldsymbol{f}\left(\boldsymbol{\Phi}^{\odot 2}\right)$. By (3.15) this implies (3.26) which leads to $\|M(g) \boldsymbol{f}\|_{\text {止 }} \leq$ $C\|g\|_{\infty}\|\boldsymbol{F}\|_{\mathbb{\sharp}}$ for a constant $C$ only depending on $m$ and $n$ which are fixed. (vii) is proved similarly to (vi) considering $\overrightarrow{\bar{\Psi}}_{+} \cdot M(G) \boldsymbol{F}\left(\boldsymbol{\Phi}_{+}^{\odot 2}, \boldsymbol{\Phi}_{-}^{\odot 2}\right) \overrightarrow{\bar{\Psi}}_{-}$. 


\section{Averages of the matrix Green's function}

We have all the main supersymmetric identities by now. So let us consider the random Hamiltonian $H_{\lambda}$ introduced in (1.1) and (1.2). Recall that we fixed some arbitrary site in $\mathbb{B}$ which we called the origin and denoted by 0 . Given two nearest neighbors sites $x, y \in \mathbb{B}$, we will denote by $\mathbb{B}^{(x \mid y)}$ the lattice obtained by removing from $\mathbb{B}$ the branch emanating from $x$ that passes through $y$; if we do not specify which branch was removed we will simply write $\mathbb{B}^{(x)}$. Each vertex in $\mathbb{B}^{(x)}$ has degree $K+1$, with the single exception of $x$ which has degree $K$. Given $\Lambda \subset \mathbb{B}$, we will use $H_{\lambda, \Lambda}$ to denote the operator $H_{\lambda}$ restricted to $\ell^{2}\left(\Lambda, \mathbb{C}^{m}\right)$ with Dirichlet boundary conditions. The matrix Green's function corresponding to $H_{\lambda, \Lambda}$ will be denoted by

$$
G_{\lambda, \Lambda}(x, y ; z)=\left[\left\langle x, j\left|\left(H_{\lambda, \Lambda}-z\right)^{-1}\right| y, k\right\rangle\right]_{j, k \in\{1, \ldots, m\}}
$$

for $x, y \in \Lambda$, and $z=E+i \eta$ with $E \in \mathbb{R}, \eta>0$.

Important choices of $\Lambda \subset \mathbb{B}$ will be the sets $\mathbb{B}_{\ell}$, denoting all sites $y \in \mathbb{B}$ with distance $|y|=d(0, y) \leq \ell$, and $\mathbb{B}_{\ell}^{(x \mid y)}$ denoting all sites $x^{\prime} \in \mathbb{B}^{(x \mid y)}$ with $d\left(x, x^{\prime}\right) \leq \ell$. We will use the Green's matrix at the origin very often, therefore let us define

$$
G_{\lambda}(z):=G_{\lambda}(0,0 ; z)
$$

For special choices of $\Lambda$ let us also introduce the following notation:

$$
\begin{aligned}
H_{\lambda, \ell} & :=H_{\lambda, \mathbb{B}_{\ell}}, & G_{\lambda, \ell}(z) & :=G_{\lambda, \mathbb{B}_{\ell}}(0,0 ; z), \\
H_{\lambda}^{(x \mid y)} & :=H_{\lambda, \mathbb{B}(x \mid y)}, & G_{\lambda}^{(x \mid y)}(z) & :=G_{\lambda, \mathbb{B}(x \mid y)}(x, x ; z), \\
H_{\lambda, \ell}^{(x \mid y)} & :=H_{\lambda, \mathbb{B}_{\ell}^{(x \mid y)}}, & G_{\lambda, \ell}^{(x \mid y)}(z) & :=G_{\lambda, \mathbb{B}_{\ell}^{(x \mid y)}}(x, x ; z), \\
H_{\lambda}^{(x)} & :=H_{\lambda, \mathbb{B}(x)}, & G_{\lambda}^{(x)}(z) & :=G_{\lambda, \mathbb{B}(x)}(x, x ; z) .
\end{aligned}
$$

Similarly to [1], Proposition 1.2, we have

$$
\left\{\begin{array}{l}
\lim _{\ell \rightarrow \infty} G_{\lambda, \mathbb{B}_{\ell}}(x, y ; z)=G_{\lambda}(x, y ; z), \\
\lim _{\ell \rightarrow \infty} G_{\lambda, \mathbb{B}_{x, \ell}}(x, y ; z)=G_{\lambda}(x, y ; z) .
\end{array}\right.
$$

To each site $x \in \mathbb{B}$ we assign supermatrices $\boldsymbol{\Phi}_{x}, \boldsymbol{\Phi}_{x,+}$, and $\boldsymbol{\Phi}_{x,-}$, which are all independent, i.e. all different Grassmann variables are independent. We will also use the independent supermatrices $\boldsymbol{\Phi}, \boldsymbol{\Phi}^{\prime}, \boldsymbol{\Phi}_{+}, \boldsymbol{\Phi}_{-}, \boldsymbol{\Phi}_{+}^{\prime}$ and $\boldsymbol{\Phi}_{-}^{\prime}$. Furthermore we may use notations like $\boldsymbol{\Phi}_{x}=\left(\boldsymbol{\varphi}_{x}, \boldsymbol{\Psi}_{x}\right)$ where $\boldsymbol{\varphi}_{x}$ is a variable varying in $\mathbb{R}^{m \times 2 n}$ and $\boldsymbol{\Psi}_{x}=\left(\left(\bar{\psi}_{x}\right)_{k, \ell},\left(\psi_{x}\right)_{k, \ell}\right)_{k, \ell}$. Also $\vec{\Psi}_{x}, \overrightarrow{\bar{\Psi}}_{x, \pm}$ and so on shall be defined analogously to $(3.1)$.

For each finite subset $\Lambda \subset \mathbb{B}$ we set $D_{\Lambda} \boldsymbol{\Phi}=\prod_{x \in \Lambda} D \boldsymbol{\Phi}_{x}$, where $D \boldsymbol{\Phi}_{x}$ is defined as in (2.9). Let $B$ be an operator on $\ell^{2}\left(\mathbb{B}, \mathbb{C}^{m}\right)$ and $B_{\Lambda}$ its restriction to $\ell^{2}\left(\Lambda, \mathbb{C}^{m}\right)$ 
for a finite set $\Lambda \subset \mathbb{B}$. For $x, y \in \Lambda$ we define $\left\langle x\left|B_{\Lambda}\right| y\right\rangle$ to be the $m \times m$ matrix with entries $\left(\left\langle x, j\left|B_{\Lambda}\right| y, k\right\rangle\right)_{j, k}$. Furthermore we define

$$
\left\langle\boldsymbol{\Phi}\left|B_{\Lambda}\right| \boldsymbol{\Phi}\right\rangle=\sum_{x, y \in \Lambda} \boldsymbol{\Phi}_{x} \cdot\left\langle x\left|B_{\Lambda}\right| y\right\rangle \boldsymbol{\Phi}_{y} .
$$

Now let $\operatorname{Im} z>0, \Lambda \subset \mathbb{B}$ finite, and $x, y \in \Lambda$. By the supersymmetric replica trick, for any replica $s \in\{1, \ldots, n\}$ we have, as in [6], [11], and [20],

$$
\left[G_{\lambda, \Lambda}(x, y ; z)\right]_{j, k}=i \int\left(\psi_{x}\right)_{j, s}\left(\bar{\psi}_{y}\right)_{k, s} e^{-i\left\langle\boldsymbol{\Phi}\left|H_{\lambda, \Lambda}-z\right| \boldsymbol{\Phi}\right\rangle} D_{\Lambda} \boldsymbol{\Phi} .
$$

For some fixed $x \in \mathbb{B}$ we will denote by $x_{0}=0, x_{1}, \ldots, x_{|x|}=x$ the shortest path from 0 to $x$, i.e. $d\left(x_{i}, x_{i-1}\right)=1$ and $x_{i} \neq x_{j}$ for $i \neq j$. We denote by $\mathbb{B}_{x, \ell}$ all sites in $\mathbb{B}$ whose distance from the path $x_{0}, \ldots, x_{|x|}$ is at most $\ell+1$. If we let $N\left(x_{i}\right)$ be the set of neighbors of $x_{i}$ which are not on the path $x_{0}, \ldots, x_{|x|}$, then, as a set,

$$
\mathbb{B}_{x, \ell}=\left\{x_{0}, x_{1} \ldots, x_{|x|}\right\} \cup\left[\bigcup_{i=0}^{|x|} \bigcup_{y \in N\left(x_{i}\right)} \mathbb{B}_{\ell}^{(y \mid x)}\right],
$$

where the union is disjoint. Note that for $|x| \geq 1$ we have $\left|N\left(x_{i}\right)\right|=K-1$ for $i=1, \ldots,|x|-1$ and $|N(0)|=|N(x)|=K$. If $|x|=0$, i.e. $x=0$, then $N(0)=K+1$.

Setting $\Lambda=\mathbb{B}_{x, \ell}$ in (4.2), noting that $\left[\vec{\Psi}_{0} \vec{\Psi}_{x}^{\top}\right]_{j, k}=\left(\psi_{0}\right)_{j, 1}\left(\bar{\psi}_{x}\right)_{k, 1}$ and using the decomposition (4.3) we obtain

$$
G_{\lambda, \mathbb{B}_{x, \ell}}(0, x ; z)=i \int \vec{\Psi}_{0} \overrightarrow{\bar{\Psi}}_{x}^{\top} \prod_{j=0}^{|x|-1} e^{-i \boldsymbol{\Phi}_{x_{j}} \cdot \boldsymbol{\Phi}_{x_{j}+1}} \prod_{j=0}^{|x|} \Theta_{j} D_{\mathbb{B}_{x, \ell}} \boldsymbol{\Phi}
$$

where

$$
\Theta_{j}=e^{i \boldsymbol{\Phi}_{x_{j}} \cdot\left(z-A-\lambda V\left(x_{j}\right)\right) \boldsymbol{\Phi}_{x_{j}}} e^{-i \sum_{y \in N\left(x_{j}\right)}\left[\boldsymbol{\Phi}_{x_{j}} \cdot \boldsymbol{\Phi}_{y}+\left\langle\boldsymbol{\Phi}\left|H_{\lambda, \ell}^{\left(y \mid x_{j}\right)}\right| \boldsymbol{\Phi}\right\rangle\right]} .
$$

In order to simplify this equation note that one obtains as in [28], eq. (3.11),

$$
\int e^{-i \boldsymbol{\Phi}_{x_{j}} \cdot \boldsymbol{\Phi}_{y}-i\left\langle\boldsymbol{\Phi}\left|H_{\lambda, \ell-1}^{\left(y \mid x_{j}\right)}-z\right| \boldsymbol{\Phi}\right\rangle} D_{\mathbb{B}_{\ell}^{\left(y \mid x_{j}\right)}} \boldsymbol{\Phi}=e^{(i / 4) \boldsymbol{\Phi}_{x_{j}} \cdot G_{\lambda, \ell}^{\left(y \mid x_{j}\right)}(z) \boldsymbol{\Phi}_{x_{j}}} .
$$

Plugging (4.6) into (4.4), using (4.1), and letting $\ell \rightarrow \infty$, we get

$$
G_{\lambda}(0, x ; z)=i \int \vec{\Psi}_{0} \overrightarrow{\bar{\Psi}}_{x}^{\top} \prod_{j=0}^{|x|-1} e^{-i \boldsymbol{\Phi}_{x_{j}} \cdot \boldsymbol{\Phi}_{x_{j+1}}} \prod_{j=0}^{|x|} \Upsilon_{\lambda, z}^{x, j}\left(\boldsymbol{\Phi}_{x_{j}}^{\odot 2}\right) \prod_{j=0}^{|x|} D \boldsymbol{\Phi}_{x_{j}},
$$

where

$$
\Upsilon_{\lambda, z}^{x, j}\left(\varphi^{\odot 2}\right)=e^{i \operatorname{Tr}\left(\left[z-\lambda V\left(x_{j}\right)-A+\frac{1}{4} \sum_{y \in N\left(x_{j}\right)} G_{\lambda}^{\left(y \mid x_{j}\right)}(z)\right] \varphi^{\odot 2}\right)} .
$$


The dependence on $x$ results from the fact that $x$ determines the path

$$
x_{0}=0, x_{1}, \ldots, x_{|x|}=x .
$$

Now we want to consider $\left|G_{\lambda}(0, x ; z)\right|^{2}$. To improve the appearance of the following equations, we introduce the following notation:

$$
\begin{gathered}
\hat{\boldsymbol{\Phi}}:=\left(\boldsymbol{\Phi}_{+}, \boldsymbol{\Phi}_{-}\right), \quad \hat{\boldsymbol{\Phi}}^{\odot 2}:=\left(\boldsymbol{\Phi}_{+}^{\odot 2}, \boldsymbol{\Phi}_{-}^{\odot 2}\right), \quad D \widehat{\boldsymbol{\Phi}}:=D \boldsymbol{\Phi}_{+} D \boldsymbol{\Phi}_{-}, \\
\hat{\boldsymbol{\varphi}}:=\left(\boldsymbol{\varphi}_{+}, \boldsymbol{\varphi}_{-}\right), \quad \hat{\boldsymbol{\varphi}}^{\odot 2}:=\left(\boldsymbol{\varphi}_{+}^{\odot 2}, \boldsymbol{\varphi}_{-}^{\odot 2}\right), \\
\hat{\boldsymbol{\Phi}}_{x}:=\left(\boldsymbol{\Phi}_{x,+}, \boldsymbol{\Phi}_{x,-}\right), \quad \hat{\boldsymbol{\Phi}}_{x}^{\odot 2}:=\left(\boldsymbol{\Phi}_{x,+}^{\odot 2}, \boldsymbol{\Phi}_{x,-}^{\odot 2}\right), \quad D \widehat{\boldsymbol{\Phi}}_{x}:=D \boldsymbol{\Phi}_{x,+} D \boldsymbol{\Phi}_{x,-}, \\
\hat{\boldsymbol{\Phi}}_{x} \cdot \hat{\boldsymbol{\Phi}}_{y}:=\boldsymbol{\Phi}_{x,+} \cdot \boldsymbol{\Phi}_{y,+}-\boldsymbol{\Phi}_{x,-} \cdot \boldsymbol{\Phi}_{y,-} .
\end{gathered}
$$

From (4.7) we obtain

$$
\begin{aligned}
\left|G_{\lambda}(0, x ; z)\right|^{2}= & G_{\lambda}^{*}(0, x ; z) G_{\lambda}(0, x ; z) \\
=- & \int \vec{\Psi}_{x,+} \vec{\Psi}_{0,+}^{\top} \Gamma_{\lambda, z}^{x, r}\left(\widehat{\boldsymbol{\Phi}}_{x}^{\odot 2}\right) \\
& \times \prod_{j=0}^{|x|-1}\left[e^{i \widehat{\boldsymbol{\Phi}}_{x_{j}}} \widehat{\boldsymbol{\Phi}}_{x_{j+1}} \Gamma_{\lambda, z}^{x, j}\left(\widehat{\boldsymbol{\Phi}}_{x_{j}}^{\odot 2}\right)\right] \vec{\Psi}_{0,-} \overrightarrow{\bar{\Psi}}_{x,-}^{\top} \prod_{j=0}^{|x|} D \widehat{\boldsymbol{\Phi}}_{x_{j}},
\end{aligned}
$$

where

$$
\Gamma_{\lambda, z}^{x, j}\left(\hat{\varphi}^{\odot 2}\right)=\bar{\Upsilon}_{\lambda, z}^{x, j}\left(\varphi_{+}^{\odot 2}\right) \Upsilon_{\lambda, z}^{x, j}\left(\varphi_{-}^{\odot 2}\right),
$$

with the bar denoting complex conjugation. The minus sign in (4.9) comes from

$$
\left[\vec{\Psi}_{0,+} \overrightarrow{\bar{\Psi}}_{x,+}^{\top}\right]^{\top}=-\overrightarrow{\bar{\Psi}}_{x,+} \vec{\Psi}_{0,+}^{\top},
$$

a consequence of the anti-commutation relations for Grassmann variables.

As in [28], for $\lambda \in \mathbb{R}, E \in \mathbb{R}$ and $\eta>0$ let us introduce $\xi_{\lambda, z} \in \mathcal{K}_{\infty}$ by

$$
\xi_{\lambda, z}\left(\hat{\varphi}^{\odot 2}\right)=\mathbb{E}\left(\exp \left\{\frac{i}{4} \operatorname{Tr}\left(G_{\lambda}^{(0)}(z) \varphi_{+}^{\odot 2}-\left[G_{\lambda}^{(0)}(z)\right]^{*} \varphi_{-}^{\odot 2}\right)\right\}\right),
$$

and the operator $\mathscr{B}_{\lambda, z}$ by

$$
\mathscr{B}_{\lambda, z}=M\left(\mathrm{e}^{i \operatorname{Tr}\left((z-A) \varphi_{+}^{\odot 2}-(\bar{z}-A) \varphi_{-}^{\odot 2}\right)} h\left(\lambda\left(\varphi_{+}^{\odot 2}-\varphi_{-}^{\odot 2}\right)\right)\right),
$$

where $M\left(g\left(\hat{\boldsymbol{\varphi}}^{\odot 2}\right)\right)$ denotes multiplication by the function $g\left(\hat{\boldsymbol{\varphi}}^{\odot 2}\right)$. The fact that $\xi_{\lambda, z} \in \mathcal{K}_{\infty}$ is a continuous family of elements is shown in [28]. $\mathscr{B}_{\lambda, z}$ and $M(g)$ act on matrix valued functions by acting on each entry. Very important will be [28], eq. (4.12) and Theorem 5.6, stating the following. 
Theorem 4.1. For $E \in I_{A, K}$ there exists $\lambda_{E}>0$ and $\varepsilon_{E}>0$, such that the continuous map

$$
\left(\lambda, E^{\prime}, \eta\right) \in\left(-\lambda_{E}, \lambda_{E}\right) \times\left(E-\varepsilon_{E}, E+\varepsilon_{E}\right) \times(0, \infty) \mapsto \xi_{\lambda, E^{\prime}+i \eta} \in \mathcal{K}_{\infty}
$$

has a continuous extension to $\left(-\lambda_{E}, \lambda_{E}\right) \times\left(E-\varepsilon_{E}, E+\varepsilon_{E}\right) \times[0, \infty)$ satisfying the fix point equation

$$
\xi_{\lambda, z}=\widehat{T} \mathcal{B}_{\lambda, z} \xi_{\lambda, z}^{K}
$$

in $\mathcal{K}_{\infty}$

We set

$$
\Xi=\left\{\bigcup_{E \in I_{A}, K}\left(-\lambda_{E}, \lambda_{E}\right) \times\left(E-\varepsilon_{E}, E+\varepsilon_{E}\right) \times[0, \infty)\right\} \cup\{\mathbb{R} \times \mathbb{R} \times(0, \infty)\},
$$

where $\lambda_{E}>0$ and $\varepsilon_{E}>0$ are as in the theorem, so we can extend $\xi_{\lambda, E+i \eta}$ to a continuous function on all of $\Xi$, defining $\xi_{\lambda, E}$ for $(\lambda, E, 0) \in \Xi$.

We further define $\xi_{\lambda, E+i \eta}$ on $\Xi$ to be the diagonal $m \times m$ matrix with $\xi_{\lambda, E+i \eta}$ on all diagonal entries, i.e.

$$
\xi_{\lambda, E+i \eta}:=\xi_{\lambda, E+i \eta} \mathbf{1}
$$

Note that the map $(\lambda, E, \eta) \mapsto \xi_{\lambda, E+i \eta} \in \mathcal{K}^{m \times m} \subset \mathbb{K}$ is also continuous on $\Xi$.

Proposition 4.2. We have

$$
\begin{aligned}
\mathbb{E}\left|G_{\lambda}(0, x ; z)\right|^{2}=-\int & \vec{\Psi}_{+} \vec{\Psi}_{+}^{\top}\left\{\mathcal{B}_{\lambda, z} M\left(\xi_{\lambda, z}^{K}\right)\right. \\
& \left.\left.\times\left[\widehat{\mathbb{T}}_{\mathcal{B}_{\lambda, z}} M\left(\xi_{\lambda, z}^{K-1}\right)\right]^{|x|} \xi_{\lambda, z}\right)\right\}\left(\hat{\boldsymbol{\Phi}}^{\odot 2}\right) \vec{\Psi}_{-} \overrightarrow{\bar{\Psi}}_{-}^{\top} D \hat{\boldsymbol{\Phi}}
\end{aligned}
$$

Proof. Let $x \neq 0 . \quad\left\{\Gamma_{\lambda, z}^{x, j}\left(\widehat{\boldsymbol{\Phi}}^{\odot 2}\right)\right\}_{j=0,1, \ldots,|x|}$ are independent Grassmann algebravalued random variables, with

$$
\mathbb{E}\left(\Gamma_{\lambda, z}^{x, j}\left(\hat{\boldsymbol{\Phi}}^{\odot 2}\right)\right)= \begin{cases}\mathcal{B}_{\lambda, z} \xi_{\lambda, z}^{K}\left(\widehat{\boldsymbol{\Phi}}^{2}\right) & \text { if } j=0 \text { or } j=|x| \\ \mathcal{B}_{\lambda, z} \xi_{\lambda, z}^{K-1}\left(\widehat{\boldsymbol{\Phi}}^{\odot 2}\right) & \text { if } 0<j<|x|\end{cases}
$$

Thus, taking expectation in (4.9) and using the matrix equality

$$
\xi_{\lambda, z}\left(\widehat{\boldsymbol{\Phi}}_{0}^{\odot 2}\right) \vec{\Psi}_{0,-} \overrightarrow{\bar{\Psi}}_{x,-}^{\top}=\xi_{\lambda, z}\left(\hat{\boldsymbol{\Phi}}_{0}^{\odot 2}\right) \mathbf{1} \vec{\Psi}_{0,-} \overrightarrow{\bar{\Psi}}_{x,-}^{\top}=\xi_{\lambda, z}\left(\widehat{\boldsymbol{\Phi}}_{0}^{\odot 2}\right) \vec{\Psi}_{0,-} \overrightarrow{\bar{\Psi}}_{x,-}^{\top},
$$

we get

$$
\begin{aligned}
& \mathbb{E}\left|G_{\lambda}(0, x ; z)\right|^{2}
\end{aligned}
$$

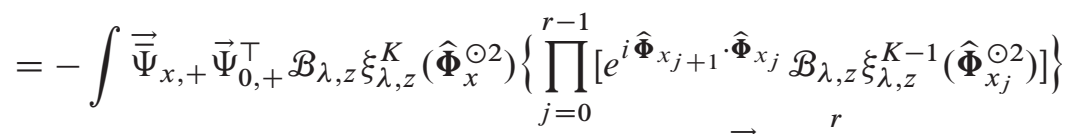

$$
\begin{aligned}
& \times \boldsymbol{\xi}_{\lambda, z}\left(\widehat{\boldsymbol{\Phi}}_{0}^{\odot 2}\right) \vec{\Psi}_{0,-} \overrightarrow{\bar{\Psi}}_{x,-}^{\top} \prod_{j=0}^{r} D \widehat{\boldsymbol{\Phi}}_{x_{j}} .
\end{aligned}
$$


Using Corollary 3.4, integration over $D \widehat{\boldsymbol{\Phi}}_{0}=D \widehat{\boldsymbol{\Phi}}_{x_{0}}$ gives

$$
\begin{aligned}
& \mathbb{E}\left|G_{\lambda}(0, x ; z)\right|^{2} \\
& =-\int \vec{\Psi}_{x,+} \vec{\Psi}_{x_{1},+}^{\top}+\mathcal{B}_{\lambda, z} \xi_{\lambda, z}^{K}\left(\hat{\boldsymbol{\Phi}}_{x}^{\odot 2}\right)\left\{\prod_{j=1}^{r-1}\left[e^{i \widehat{\boldsymbol{\Phi}}_{x_{j+1}} \cdot \widehat{\boldsymbol{\Phi}}_{x_{j}}} \mathcal{B}_{\lambda, z} \xi_{\lambda, z}^{K-1}\left(\hat{\boldsymbol{\Phi}}_{x_{j}}^{\odot 2}\right)\right]\right\} \\
& \quad \times\left[\hat{\mathbb{T}} \mathcal{B}_{\lambda, z} M\left(\xi_{\lambda, z}^{K-1}\right) \boldsymbol{\xi}_{\lambda, z}\right]\left(\hat{\boldsymbol{\Phi}}_{x_{1}}^{\odot 2}\right) \vec{\Psi}_{x_{1},-} \vec{\Psi}_{x,-}^{\top} \prod_{j=0}^{r} D \widehat{\boldsymbol{\Phi}}_{x_{j}} .
\end{aligned}
$$

Repeated similar integrations over $D \widehat{\boldsymbol{\Phi}}_{x_{j}}$ for $j=1,2, \ldots, r-1$, yields (4.12) after renaming $\widehat{\boldsymbol{\Phi}}_{x}=\widehat{\boldsymbol{\Phi}}_{x_{|x|}}$ as $\hat{\boldsymbol{\Phi}}$.

For the case $x=0$ note that $\mathbb{E}\left(\Gamma_{\lambda, z}^{0,0}\left(\hat{\boldsymbol{\Phi}}^{\odot 2}\right)\right)=\mathscr{B}_{\lambda, z} \xi_{\lambda, z}^{K+1}\left(\hat{\boldsymbol{\Phi}}^{\odot 2}\right)$, which gives (4.12) also for $x=0$.

To write the trace of $\left|G_{\lambda}(0, x ; z)\right|^{2}$ in a more compact way, let us introduce the following notations. First let us define the operator

$$
\mathcal{V}_{\lambda, z}:=\hat{\mathbb{T}} \mathscr{B}_{\lambda, z} M\left(\xi_{\lambda, z}^{K-1}\right) .
$$

Note that $\mathcal{V}_{\lambda, z}$ is a bounded linear operator on $\mathbb{K}$ in view of Proposition 3.5 (vii). For $\boldsymbol{F}, \boldsymbol{F}^{\prime} \in \mathbb{K}$ we define the bilinear forms

$$
\begin{aligned}
\left.\left\langle\boldsymbol{F} \mid \boldsymbol{F}^{\prime}\right\rangle\right\rangle: & =-\operatorname{Tr}\left\{\int \boldsymbol{F}^{\top}\left(\hat{\boldsymbol{\Phi}}^{\odot 2}\right) \overrightarrow{\bar{\Psi}}_{+} \vec{\Psi}_{+}^{\top} \boldsymbol{F}^{\prime}\left(\hat{\boldsymbol{\Phi}}^{\odot 2}\right) \vec{\Psi}_{-} \overrightarrow{\bar{\Psi}}_{-}^{\top} D \widehat{\boldsymbol{\Phi}}\right\} \\
& =-\int\left[\overrightarrow{\bar{\Psi}}_{+} \cdot \boldsymbol{F}\left(\hat{\boldsymbol{\Phi}}^{\odot 2}\right) \overrightarrow{\bar{\Psi}}_{-}\right]\left[\vec{\Psi}_{+} \cdot \boldsymbol{F}^{\prime}\left(\hat{\boldsymbol{\Phi}}^{\odot 2}\right) \vec{\Psi}_{-}\right] D \widehat{\boldsymbol{\Phi}},
\end{aligned}
$$

and

$$
\left.\left\langle\boldsymbol{F} \mid \boldsymbol{F}^{\prime}\right\rangle\right\rangle_{\lambda, z}:=\left\langle\mathscr{B}_{\lambda, z} M\left(\xi_{\lambda, z}^{K-1}\right) \boldsymbol{F} \mid \boldsymbol{F}^{\prime}\right\rangle=\left\langle\left\langle\boldsymbol{F} \mid \mathscr{B}_{\lambda, z} M\left(\xi_{\lambda, z}^{K-1}\right) \boldsymbol{F}^{\prime}\right\rangle .\right.
$$

For the second equation in (4.15), note that for matrices $\boldsymbol{F}, \boldsymbol{F}^{\prime}$ whose entries are even elements of the Grassmann algebra $\Lambda\left(\boldsymbol{\Psi}_{+}, \boldsymbol{\Psi}_{-}\right)$, we have

$$
\begin{aligned}
\operatorname{Tr}\left\{\boldsymbol{F}^{\top} \overrightarrow{\bar{\Psi}}_{+} \vec{\Psi}_{+}^{\top} \boldsymbol{F}^{\prime}\left(\hat{\boldsymbol{\Phi}}^{\odot 2}\right) \vec{\Psi}_{-} \overrightarrow{\bar{\Psi}}_{-}^{\top}\right\} & =-\left[\overrightarrow{\bar{\Psi}}_{-} \cdot \boldsymbol{F}^{\top} \overrightarrow{\bar{\Psi}}_{+}\right]\left[\vec{\Psi}_{+} \cdot \boldsymbol{F}^{\prime} \vec{\Psi}_{-}\right] \\
& =\left[\overrightarrow{\bar{\Psi}}_{+} \cdot \boldsymbol{F} \overrightarrow{\bar{\Psi}}_{-}\right]\left[\vec{\Psi}_{+} \cdot \boldsymbol{F}^{\prime} \vec{\Psi}_{-}\right]
\end{aligned}
$$

The sign changes are caused by the anti-commutation relations of the Grassmann variables.

Proposition 4.3. The following identities hold.

$$
\begin{aligned}
\left\langle\boldsymbol{F} \mid \hat{\mathbb{T}} \boldsymbol{F}^{\prime}\right\rangle & =\left\langle\left\langle\hat{\mathbb{V}} \boldsymbol{F} \mid \boldsymbol{F}^{\prime}\right\rangle,\right. \\
\left.\left\langle\boldsymbol{F} \mid \mathcal{V}_{\lambda, z} \boldsymbol{F}^{\prime}\right\rangle\right\rangle_{\lambda, z} & =\left\langle\left\langle\mathcal{V}_{\lambda, z} \boldsymbol{F} \mid \boldsymbol{F}^{\prime}\right\rangle_{\lambda, z},\right. \\
\mathbb{E}\left(\operatorname{Tr}\left(\left|G_{\lambda}(0, x ; z)\right|^{2}\right)\right) & =\left\langle\left\langle\xi_{\lambda, z} \mid \mathcal{V}_{\lambda, z}^{|x|} \xi_{\lambda, z}\right\rangle_{\lambda, z} .\right.
\end{aligned}
$$


Proof. Using Corollary 3.4 and (4.15) we get

$$
\begin{aligned}
& \left\langle\boldsymbol{F} \mid \hat{\mathbb{T}} \boldsymbol{F}^{\prime}\right\rangle=-\int\left[\overrightarrow{\bar{\Psi}}_{+} \cdot \boldsymbol{F}\left(\hat{\boldsymbol{\Phi}}^{\odot 2}\right) \overrightarrow{\bar{\Psi}}_{-}\right] e^{i \hat{\boldsymbol{\Phi}} \cdot \hat{\boldsymbol{\Phi}}^{\prime}}\left[\vec{\Psi}_{+}^{\prime} \cdot \boldsymbol{F}^{\prime}\left(\hat{\boldsymbol{\Phi}}^{\prime \odot 2}\right) \vec{\Psi}_{-}^{\prime}\right] D \widehat{\boldsymbol{\Phi}}^{\prime} D \hat{\boldsymbol{\Phi}} \\
& =-\int\left[\vec{\Psi}_{+}^{\prime} \cdot \hat{\mathbb{T}} \boldsymbol{F}\left(\hat{\boldsymbol{\Phi}}^{\prime \odot 2}\right){\overrightarrow{\bar{\Psi}^{\prime}}}_{-}\right]\left[\vec{\Psi}_{+}^{\prime} \cdot \boldsymbol{F}^{\prime}\left(\widehat{\boldsymbol{\Phi}}^{\prime \odot 2}\right) \vec{\Psi}_{-}^{\prime}\right] D \widehat{\boldsymbol{\Phi}}^{\prime} \\
& =\left\langle\hat{\mathbb{T}} \boldsymbol{F} \mid \boldsymbol{F}^{\prime}\right\rangle \text {. }
\end{aligned}
$$

Eq. (4.18) is now a consequence of (4.16) and (4.17); eq. (4.19) follows from (4.12), (4.15) and (4.16).

Similarly to [24], for $z=E+i \eta$ with $\eta>0$ we define $\boldsymbol{\theta}_{\lambda, z} \in \mathbb{K}$ by

$$
\boldsymbol{\theta}_{\lambda, z}:=-2(\partial \otimes \mathbf{1}+\mathbf{1} \otimes \partial) \xi_{\lambda,, z}=-2\left(\partial_{+}+\partial_{-}\right) \xi_{\lambda, z} .
$$

Using $\xi_{\lambda, z}=\xi_{\lambda, z}^{\top}$ and $\partial_{-} \xi_{\lambda, z}=\left[\partial_{-} \xi_{\lambda, z}\right]^{\top}$, the second equation follows from (3.23) and (3.24). Proposition 3.5 (iv) implies that the map $(\lambda, E, \eta) \mapsto$ $\boldsymbol{\theta}_{\lambda, E+i \eta} \in \mathbb{K}$ is continuous on $\boldsymbol{\Xi}$.

Lemma 4.4. For $\eta=\operatorname{Im} z>0$ we have

$$
\begin{aligned}
\left.\left\langle\boldsymbol{\theta}_{\lambda, z} \mid \mathcal{V}_{\lambda, z}^{|x|} \xi_{\lambda, z}\right\rangle\right\rangle_{\lambda, z} & =\mathbb{E}\left(\operatorname{Tr}\left(\Im_{\lambda}^{\left(x^{\prime} \mid x\right)}(z)\left|G_{\lambda}(0, x ; z)\right|^{2}\right)\right) \\
& =\mathbb{E}\left(\operatorname{Tr}\left(\left|G_{\lambda}(0, x ; z) \sqrt{\widetilde{\Im}_{\lambda}^{\left(x^{\prime} \mid x\right)}(z)}\right|^{2}\right)\right)>0,
\end{aligned}
$$

and

$$
\begin{aligned}
& \left\langle\boldsymbol{\theta}_{\lambda, z} \mid \mathcal{V}_{\lambda, z}^{|x|} \boldsymbol{\theta}_{\lambda, z}\right\rangle_{\lambda, z} \\
& =\mathbb{E}\left(\operatorname{Tr}\left(\Im_{\lambda}^{\left(x^{\prime} \mid x\right)}(z) G_{\lambda}^{*}(0, x ; z) \Im_{\lambda}^{\left(0^{\prime} \mid 0\right)}(z) G_{\lambda}(0, x ; z)\right)\right) \\
& =\mathbb{E}\left(\operatorname{Tr}\left(\left|\sqrt{\Im_{\lambda}^{\left(x^{\prime} \mid x\right)}(z)} G_{\lambda}(0, x ; z) \sqrt{\Im_{\lambda}^{\left(0^{\prime} \mid 0\right)}(z)}\right|^{2}\right)\right)>0,
\end{aligned}
$$

where $0^{\prime} \in \mathbb{B}$ is a neighbor of $0 \in \mathbb{B}$ and $x^{\prime} \in \mathbb{B}$ is a neighbor of $x \in \mathbb{B}$, both not lying on the path from 0 to $x$.

Proof. As $H_{\lambda}$ is a real operator, the Green's matrices $G_{\lambda}^{(x \mid y)}(z)$ are symmetric, implying $\left[G_{\lambda}^{(x \mid y)}(z)\right]^{*}=\overline{G_{\lambda}^{(x \mid y)}(z)}$, where the overline denotes complex conjugation. It follows that the imaginary parts $\Im_{\lambda}^{(x \mid y)}(z):=\frac{1}{2 i}\left(G_{\lambda}^{(x \mid y)}(z)-\left[G_{\lambda}^{(x \mid y)}(z)\right]^{*}\right)$ are real, symmetric matrices. More over, $\mathfrak{\Im}_{\lambda}^{(x \mid y)}$ is positive if $\eta=\operatorname{Im} z>0$. Since

$$
\partial e^{\operatorname{Tr}(M \varphi \odot 2)}=M e^{\operatorname{Tr}(M \varphi \odot 2)},
$$

for symmetric $m \times m$ matrices $M$, we obtain

$$
\boldsymbol{\theta}_{\lambda, z}\left(\hat{\boldsymbol{\varphi}}^{\odot 2}\right)=\mathbb{E}\left(\Im_{\lambda}^{(0)}(z) \exp \left\{\frac{i}{4} \operatorname{Tr}\left(G_{\lambda}^{(0)}(z) \boldsymbol{\varphi}_{+}^{\odot 2}-\left[G_{\lambda}^{(0)}(z)\right]^{*} \boldsymbol{\varphi}_{-}^{\odot 2}\right)\right\}\right) .
$$


In particular, $\boldsymbol{\theta}_{\lambda, z}$ is a symmetric matrix. Eq. (4.24) implies

$$
\mathscr{B}_{\lambda, z} M\left(\xi_{\lambda, z}^{K-1}\right) \boldsymbol{\theta}_{\lambda, z}=\mathbb{E}\left(\Im_{\lambda}^{\left(x^{\prime} \mid x\right)}(z) \Gamma_{\lambda, z}^{x,|x|}\right)=\mathbb{E}\left(\Im_{\lambda}^{\left(0^{\prime} \mid 0\right)}(z) \Gamma_{\lambda, z}^{x, 0}\right) .
$$

Multiplying (4.9) by $\Im_{\lambda}^{\left(x^{\prime} \mid x\right)}(z)$ from the left, taking expectations and combining this with Corollary 3.4, equations (4.15), (4.16), (4.25), and the fact that $\boldsymbol{\theta}_{\lambda, z}^{\top}=\boldsymbol{\theta}_{\lambda, z}$ gives the first equation in (4.21). To get the first equation in (4.22) we multiply (4.9) by $\Im_{\lambda}^{\left(x^{\prime} \mid x\right)}(z)$ from the left, insert the matrix $\Im_{\lambda}^{\left(0^{\prime} \mid 0\right)}(z)$ between the matrices $\vec{\Psi}_{x,+} \vec{\Psi}_{0,+}^{\top}$ and $\vec{\Psi}_{0,-} \overrightarrow{\bar{\Psi}}_{x,-}^{\top}$, and take expectations.

The only thing left to prove are the inequalities in (4.21) and (4.22). Since $\Im_{\lambda}^{\left(0^{\prime} \mid 0\right)}(z)$ and $\Im_{\lambda}^{\left(x^{\prime} \mid x\right)}(z)$ are both invertible, both inequalities will follow if we can show that the matrix $G_{\lambda}(0, x ; z)$ is not identically zero for almost all potentials.

Let $H_{\lambda}^{\left(0 \neq x_{1}\right)}=H_{\lambda}^{\left(0 \mid x_{1}\right)} \oplus H_{\lambda}^{\left(x_{1} \mid 0\right)}$, then $H_{\lambda}=H_{\lambda}^{\left(0 \neq x_{1}\right)}+\Gamma$ where

$$
\langle y, k|\Gamma| z, j\rangle=\frac{1}{2}\left(\delta_{y, 0} \delta_{j, k} \delta_{z, x_{1}}+\delta_{y, x_{1}} \delta_{j, k} \delta_{z, 0}\right) .
$$

Using the resolvent identity,

$$
\left(H_{\lambda}-z\right)^{-1}=\left(H_{\lambda}^{\left(0+x_{1}\right)}-z\right)^{-1}-\left(H_{\lambda}^{\left(0+x_{1}\right)}-z\right)^{-1} \Gamma\left(H_{\lambda}-z\right)^{-1},
$$

and the fact that $\left\langle 0\left|\left(H_{\lambda}^{\left(0 \nmid x_{1}\right)}-z\right)^{-1}\right| x\right\rangle=0$, we obtain the matrix equation

$$
G_{\lambda}(0, x ; z)=-\frac{1}{2} G_{\lambda}^{\left(0 \mid x_{1}\right)}(0,0 ; z) G_{\lambda}\left(x_{1}, x ; z\right) .
$$

Iterating this procedure gives

$$
G_{\lambda}(0, x ; z)=\left(-\frac{1}{2}\right)^{|x|}\left[\prod_{j=1}^{|x|} G_{\lambda}^{\left(x_{j-1} \mid x_{j}\right)}(z)\right] G_{\lambda}(x, x ; z) .
$$

For $\eta=\operatorname{Im} z>0$ the imaginary parts of these matrix Green's functions on the right hand side are positive. Therefore all these matrices are invertible and hence $G_{\lambda}(0, x ; z)$ is invertible and hence not zero (for all random potentials).

\section{The proof of Theorem 1.2}

From now on the proof is completely analogous to [24]. By (4.19) we obtain

$$
J_{\lambda}(z):=\sum_{x \in \mathbb{B}}|x|^{2} \mathbb{E}\left(\operatorname{Tr}\left(\left|G_{\lambda}(0, x ; z)\right|^{2}\right)\right)=\frac{K+1}{K} \sum_{r=1}^{\infty} r^{2}\left\langle\left\langle\xi_{\lambda, z} \mid \mathcal{W}_{\lambda, z}^{r} \xi_{\lambda, z}\right\rangle_{\lambda, z},\right.
$$

where $\mathcal{W}_{\lambda, z}=K \mathcal{V}_{\lambda, z}$ and $\operatorname{Im} z=\eta>0$. 
Lemma 5.1. Let $\lambda \in \mathbb{R}, z=E+i \eta$ with $E \in \mathbb{R}$ and $\eta>0$.

(i) For all $\boldsymbol{F}, \boldsymbol{F}^{\prime} \in \mathbb{K}$

$$
\left\langle\boldsymbol{F} \mid \mathcal{W}_{\lambda, z} \boldsymbol{F}^{\prime}\right\rangle_{\lambda, z}=\left\langle\left\langle\mathcal{W}_{\lambda, z} \boldsymbol{F} \mid \boldsymbol{F}^{\prime}\right\rangle_{\lambda, z}\right.
$$

(ii) $W_{\lambda, z}^{2}$ is a compact operator on $\mathbb{K}$.

(iii) We have

$$
\mathcal{W}_{\lambda, z} \boldsymbol{\theta}_{\lambda, z}=\boldsymbol{\theta}_{\lambda, z}-\frac{4 \eta}{K} \mathcal{W}_{\lambda, z} \xi_{\lambda, z}
$$

(iv) For any $r=0,1,2, \ldots$ we have

$$
\left\langle\left\langle\boldsymbol{\xi}_{\lambda, z} \mid \mathcal{W}_{\lambda, z}^{r} \boldsymbol{\theta}_{\lambda, z}\right\rangle_{\lambda, z}>\left\langle\left\langle\boldsymbol{\xi}_{\lambda, z} \mid \mathcal{W}_{\lambda, z}^{r+1} \boldsymbol{\theta}_{\lambda, z}\right\rangle_{\lambda, z}>0\right.\right.
$$

and

$$
\left\langle\left\langle\boldsymbol{\theta}_{\lambda, z} \mid \mathcal{W}_{\lambda, z}^{r} \boldsymbol{\theta}_{\lambda, z}\right\rangle_{\lambda, z}>\left\langle\left\langle\boldsymbol{\theta}_{\lambda, z} \mid \mathcal{W}_{\lambda, z}^{r+1} \boldsymbol{\theta}_{\lambda, z}\right\rangle_{\lambda, z}>0\right.\right.
$$

Proof. (i) follows from (4.18). (ii) is a consequence of $\mathscr{B}_{\lambda, z} M\left(\xi_{\lambda, z}^{K-1}\right) \hat{\mathbb{T}} \mathscr{B}_{\lambda, z}$ being a compact operator on $\mathbb{K}$ for $\eta>0$, which can be shown analogously to [28], Lemma 5.1(i), using (3.17) as well as the the Leibniz rules (2.19) and (3.26).

To prove (iii), note first that by $(2.25),(2.26)$ and (4.11) we have

$$
\begin{aligned}
& \boldsymbol{\theta}_{\lambda, z}=-2(\boldsymbol{\partial} \otimes \mathbf{1}+\mathbf{1} \otimes \boldsymbol{\partial}) \widehat{T} \mathscr{B}_{\lambda, z} \boldsymbol{\xi}_{\lambda, z}^{K} \\
& =(-2)^{2 m n-1}\left(\mathcal{F} \delta^{n-1} \boldsymbol{\gamma} \otimes \mathscr{F} \boldsymbol{\delta}^{n}+\mathcal{F} \boldsymbol{\delta}^{n} \otimes \mathcal{F} \boldsymbol{\delta}^{n-1} \boldsymbol{\phi}\right) \mathcal{B}_{\lambda, z} \xi_{\lambda, z}^{K} \\
& =(-2)^{2 m n-1}\left(\mathcal{F} \delta^{n-1} \boldsymbol{\gamma} \otimes \mathscr{F} \boldsymbol{\delta}^{n-1} \boldsymbol{\gamma}\right)(\mathbf{1} \otimes \boldsymbol{\partial}+\boldsymbol{\partial} \otimes \mathbf{1}) \mathcal{B}_{\lambda, z} \boldsymbol{\xi}_{\lambda, z}^{K} \\
& =-2 \hat{\mathbb{T}}\left[\left(\partial_{+}+\partial_{-}\right) B_{\lambda, z} \xi_{\lambda, z}^{K}\right] \text {. }
\end{aligned}
$$

Using (4.23) leads to

$$
\begin{array}{r}
-2\left(\partial_{+}+\partial_{-}\right)\left(e^{i \operatorname{Tr}\left((z-A) \varphi_{+}^{\odot 2}-(\bar{z}-A) \varphi \bigodot^{\odot}\right)} h\left(\lambda\left(\varphi_{+}^{\odot 2}-\varphi_{-}^{\odot 2}\right)\right)\right) \\
=4 \eta\left(e^{i \operatorname{Tr}\left((z-A) \varphi_{+}^{\odot 2}-(\bar{z}-A) \varphi_{-}^{\odot 2}\right)} h\left(\lambda\left(\varphi_{+}^{\odot 2}-\varphi_{-}^{\odot 2}\right)\right)\right),
\end{array}
$$

which combined with (5.1) gives

$$
\boldsymbol{\theta}_{\lambda, z}=-2 \widehat{\mathbb{T}}\left[\left(\partial_{+}+\boldsymbol{\partial}_{-}\right) \mathcal{B}_{\lambda, z} \boldsymbol{\xi}_{\lambda, z}^{K}\right]=\mathcal{W}_{\lambda, z} \boldsymbol{\theta}_{\lambda, z}+\frac{4 \eta}{K} \mathcal{W}_{\lambda, z} \boldsymbol{\xi}_{\lambda, z}
$$

The second inequalities in (5.1) and (5.1) follow from (4.21) and (4.22). Using (5.1), we have

$$
\begin{aligned}
\left\langle\left\langle\xi_{\lambda, z} \mid \mathcal{W}_{\lambda, z}^{r+1} \boldsymbol{\theta}_{\lambda, z}\right\rangle_{\lambda, z}\right. & =\left\langle\left\langle\xi_{\lambda, z} \mid \mathcal{W}_{\lambda, z}^{r} \boldsymbol{\theta}_{\lambda, z}\right\rangle_{\lambda, z}-\frac{4 \eta}{K}\left\langle\xi_{\lambda, z} \mid \mathcal{W}_{\lambda, z}^{r+1} \xi_{\lambda, z}\right\rangle_{\lambda, z}\right. \\
& <\left\langle\left\langle\xi_{\lambda, z} \mid \mathcal{W}_{\lambda, z}^{r} \boldsymbol{\theta}_{\lambda, z}\right\rangle_{\lambda, z},\right.
\end{aligned}
$$


since $\left\langle\left\langle\xi_{\lambda, z} \mid \mathcal{W}_{\lambda, z}^{r+1} \xi_{\lambda, z}\right\rangle_{\lambda, z}>0\right.$ by (4.19). Similarly,

$$
\begin{aligned}
\left\langle\boldsymbol{\theta}_{\lambda, z} \mid \mathcal{W}_{\lambda, z}^{r+1} \boldsymbol{\theta}_{\lambda, z}\right\rangle_{\lambda, z} & =\left\langle\left\langle\boldsymbol{\theta}_{\lambda, z} \mid \mathcal{W}_{\lambda, z}^{r} \boldsymbol{\theta}_{\lambda, z}\right\rangle_{\lambda, z}-\frac{4 \eta}{K}\left\langle\boldsymbol{\theta}_{\lambda, z} \mid \mathcal{W}_{\lambda, z}^{r+1} \xi_{\lambda, z}\right\rangle_{\lambda, z}\right. \\
& <\left\langle\left\langle\boldsymbol{\theta}_{\lambda, z} \mid \mathcal{W}_{\lambda, z}^{r} \boldsymbol{\theta}_{\lambda, z}\right\rangle_{\lambda, z},\right.
\end{aligned}
$$

since $\left\langle\left\langle\boldsymbol{\theta}_{\lambda, z} \mid \mathcal{W}_{\lambda, z}^{r+1} \xi_{\lambda, z}\right\rangle_{\lambda, z}>0\right.$ by (4.21). Thus (iv) is proven.

This lemma is just the generalization of Lemma 4.1 in [24] to the Bethe strip. Thus, from this point on we can use the exact same arguments as in [24], Lemmata 4.2-4.4, to finally obtain

$$
\begin{gathered}
J_{\lambda}(E+i \eta) \geq \frac{K+1}{4 \eta}\left\langle\left\langle\boldsymbol{\xi}_{\lambda, z} \mid \boldsymbol{\theta}_{\lambda, z}\right\rangle_{\lambda, z}+\frac{3 K(K+1)}{16 \eta^{2}}\left\langle\boldsymbol{\theta}_{\lambda, z} \mid \boldsymbol{\theta}_{\lambda, z}\right\rangle_{\lambda, z}\right. \\
+\frac{K^{2}(K+1)}{64 \eta^{3}} \frac{\left(\left\langle\boldsymbol{\theta}_{\lambda, z} \mid \boldsymbol{\theta}_{\lambda, z}\right\rangle_{\lambda, z}-\frac{4 \eta}{K}\left\langle\left\langle\boldsymbol{\xi}_{\lambda, z} \mid \boldsymbol{\theta}_{\lambda, z}\right\rangle_{\lambda, z}\right)^{2}\right.}{\left\langle\left\langle\boldsymbol{\xi}_{\lambda, z} \mid \boldsymbol{\theta}_{\lambda, z}\right\rangle_{\lambda, z}\right.}
\end{gathered}
$$

for $\lambda \in \mathbb{R}, z=E+i \eta$ with $E \in \mathbb{R}$ and $\eta>0$.

In order to do perturbation theory we have to compute some of the expressions for $\lambda=0$ and $\eta=0$ first. For an energy $E \in I_{A, K}$ we obtain from [28], eq. (3.19) and eq. (4.7), the limit as $\eta \downarrow 0$ of $\xi_{0, E+i \eta}$ (point wise and in $\mathcal{K}_{\infty}$ ), given by

$$
\xi_{0, E}\left(\varphi_{+}^{\odot 2}, \varphi_{-}^{\odot 2}\right)=e^{-i \operatorname{Tr}\left(A_{E} \varphi_{+}^{\odot 2}-\bar{A}_{E} \varphi{ }^{\odot 2}\right)},
$$

where $A_{E}$ is the matrix

$$
A_{E}=\frac{1}{2 K}\left((E-A)-i \sqrt{K-(E-A)^{2}}\right) .
$$

Here we identify numbers with multiples of the unit $m \times m$ matrix. Note that $E \in I_{A, K}$ is equivalent to $-\sqrt{K}<E-A<\sqrt{K}$ in the sense of matrices, and for such energies we get

$$
\boldsymbol{\theta}_{0, E}=-2\left(\partial_{+}+\partial_{-}\right) \xi_{0, E}=\frac{2 \sqrt{K-(E-A)^{2}}}{K} \xi_{0, E}
$$

Thus

$$
\begin{aligned}
\left\langle\boldsymbol{\xi}_{0, E} \mid \boldsymbol{\xi}_{0, E}\right\rangle_{0, E} & =\mathbb{E}\left(\operatorname{Tr}\left(\left|G_{0}(0,0 ; E)\right|^{2}\right)\right) \\
& =\operatorname{Tr}\left(4 K\left[(K+1)^{2}-4(E-A)^{2}\right]^{-1}\right)>\frac{4 m K}{(K-1)^{2}},
\end{aligned}
$$

and we get

$$
\begin{aligned}
& \left\langle\left\langle\boldsymbol{\xi}_{0, E} \mid \boldsymbol{\theta}_{0, E}\right\rangle_{0, E}\right. \\
& \quad=\operatorname{Tr}\left(\left[8 \sqrt{K-(E-A)^{2}}\right]\left[(K+1)^{2}-4(E-A)^{2}\right]^{-1}\right)>0,
\end{aligned}
$$

and

$$
\begin{aligned}
& \left\langle\boldsymbol{\theta}_{0, E} \mid \boldsymbol{\theta}_{0, E}\right\rangle_{0, E} \\
& \quad=\operatorname{Tr}\left(\left[16\left(K-(E-A)^{2}\right)\right]\left[K\left((K+1)^{2}-4(E-A)^{2}\right)\right]^{-1}\right)>0 .
\end{aligned}
$$

We can finally prove Theorem 1.2. 
Proof of Theorem 1.2. We only need to prove (1.2). Recall that the maps $(\lambda, E, \eta) \rightarrow$ $\xi_{\lambda, E+i \eta} \in \mathcal{K}_{\infty},(\lambda, E, \eta) \mapsto \xi_{\lambda, E+i \eta} \in \mathbb{K}$ and $(\lambda, E, \eta) \mapsto \boldsymbol{\theta}_{\lambda, E+i \eta} \in \mathbb{K}$ are continuous on $\Xi$, which by construction is an open neighborhood of $\{(0, E, 0): E \in$ $\left.I_{A, K}\right\}$ in $\mathbb{R} \times \mathbb{R} \times[0, \infty)$. Using (3.26) and Dominated Convergence one obtains that the $\operatorname{map}(\lambda, E, \eta) \in \mathbb{R} \times \mathbb{R} \times[0, \infty) \mapsto \mathcal{B}_{\lambda, E+i \eta} \in B(\mathbb{K})$ is continuous with respect to the strong operator topology. By Proposition 3.5 we conclude that the map $(\lambda, E, \eta) \in$ $\Xi \rightarrow B_{\lambda, E+i \eta} M\left(\xi_{\lambda, E+i \eta}^{K-1}\right) \boldsymbol{\theta}_{\lambda, E+i \eta} \in \mathbb{K}$ is continuous. Thus, it follows from (4.16), the definition of $\langle\cdot \mid \cdot \cdot\rangle_{\lambda, z}$, that the real valued maps (cf Lemma 4.4) $(\lambda, E, \eta) \rightarrow$ $\left\langle\left\langle\boldsymbol{\xi}_{\lambda, E+i \eta} \mid \boldsymbol{\theta}_{\lambda, E+i \eta}\right\rangle_{\lambda, E+i \eta}\right.$ and $(\lambda, E, \eta) \rightarrow\left\langle\left\langle\boldsymbol{\theta}_{\lambda, E+i \eta} \mid \boldsymbol{\theta}_{\lambda, E+i \eta}\right\rangle_{\lambda, E+i \eta}\right.$ have continuous extensions to $\Xi$. Moreover, by (5.3) and (5.4) these extensions satisfy

$$
\left\langle\langle \boldsymbol { \xi } _ { \lambda , E + i \eta } | \boldsymbol { \theta } _ { \lambda , E + i \eta } \rangle _ { \lambda , E + i \eta } > 0 \text { and } \left\langle\left\langle\boldsymbol{\theta}_{\lambda, E+i \eta} \mid \boldsymbol{\theta}_{\lambda, E+i \eta}\right\rangle_{\lambda, E+i \eta}>0\right.\right. \text {, }
$$

for $(\lambda, E, \eta)$ in some open neighborhood of $\left\{(0, E, 0): E \in I_{A, K}\right\}$ in $\mathbb{R} \times \mathbb{R} \times[0, \infty)$. Eq. (1.2) now follows from (5.2).

\section{References}

[1] V. Acosta and A. Klein, Analyticity of the density of states in the Anderson model in the Bethe lattice. J. Stat. Phys. 69 (1992), 277-305. MR 1184777 Zbl 0893.60093

[2] M.Aizenman, Localization at weak disorder: some elementary bounds. Rev. Math. Phys. 6 (1994), 1163-1182. Zbl 0843.47039

[3] M. Aizenman and S. Molchanov, Localization at large disorder and extreme energies: an elementary derivation. Commun. Math. Phys. 157 (1993), 245-278. MR 1244867 Zbl 0782.60044

[4] M. Aizenman, R. Sims, and S. Warzel, Stability of the absolutely continuous spectrum of random Schrödinger operators on tree graphs. Prob. Theor. Relat. Fields 136 (2006), 363-394. MR 2257129 Zbl 1169.82007

[5] M. Aizenman and S. Warzel, Resonant delocalization for random Schrödinger operators on trees. Preprint 2011. arXiv:1104.0969

[6] F. A. Berezin, The method of second quantization. Academic Press, New York and London, 1966. MR $0208930 \mathrm{Zbl} 0151.44001$

[7] M. Campanino and A. Klein, A supersymmetric transfer matrix and differentiability of the density of states in the one-dimensional Anderson model. Commun. Math. Phys. 104 (1986), 227-241. MR 0836001 Zbl 0603.60061

[8] R. Carmona, A. Klein, and F. Martinelli, Anderson localization for Bernoulli and other singular potentials. Commun. Math. Phys. 108(1987), 41-66. MR 0872140Zbl 0615.60098

[9] F. Delyon, Y. Levy, and B. Souillard, Anderson localization for multidimensional systems at large disorder or low energy. Commun. Math. Phys. 100 (1985), 463-470. MR 0806247 Zbl 0576.60053

[10] H. von Dreifus and A. Klein, A new proof of localization in the Anderson tight binding model. Commun. Math. Phys. 124 (1989), 285-299. MR 1012868 Zbl 0698.60051 
[11] K. B. Efetov, Supersymmetry and theory of disordered metals. Advances Phys. 32 (1983), 53-127. MR 0708812

[12] R. Froese, F. Halasan, and D. Hasler, Absolutely continuous spectrum for the Anderson model on a product of a tree with a finite graph. Preprint 2010. arXiv:1008.2949v1

[13] R. Froese, D. Hasler, and W. Spitzer, Absolutely continuous spectrum for the Anderson Model on a tree: a geometric proof of Klein's Theorem. Commun. Math. Phys. 269 (2007), 239-257. MR 2274470 Zbl 1117.82024

[14] R. Froese, D. Hasler, and W. Spitzer, Absolutely continuous spectrum for a random potential on a tree with strong transverse correlations and large weighted loops. Rev. Math. Phys. 21 (2009), 709-733. MR 2547606 Zbl 1168.82315

[15] J. Fröhlich, F. Martinelli, E. Scoppola, and T. Spencer Constructive proof of localization in the Anderson tight binding model, Commun. Math. Phys. 101 (1985), 21-46. MR 0814541 Zbl 0573.60096

[16] J. Fröhlich and T. Spencer, Absence of diffusion in the Anderson tight binding model for large disorder or low energy, Commun. Math. Phys. 88 (1983), 151-184. MR 0696803 Zbl 0519.60066

[17] I. Ya. Gol'dsheid, S. A. Molchanov, and L. A. Pastur, Pure point spectrum of stochastic one dimensional Schrödinger operators, Funct. Anal. Appl. 11 (1977), 1-10. MR 0470515 Zbl 0368.34015

[18] F. Halasan, Absolutely continuous spectrum for the Anderson model on some tree-like graphs. Preprint 2008. arXiv:0810.2516v3

[19] M. Keller, D. Lenz, and S. Warzel, On the spectral theory of trees with finite forward cone type. Preprint 2009. arXiv:1001.3600v1

[20] A. Klein, The supersymmetric replica trick and smoothness of the density of states for random Schrodinger operators. Proc. Symp. in Pure Mathem. 51 (1990), 315-331. MR 1077393 Zbl 0709.60105

[21] A. Klein, Localization in the Anderson model with long range hopping. Braz. J. Phys. 23 (1993), 363-371.

[22] A. Klein, Absolutely continuous spectrum in the Anderson model on the Bethe lattice. Math. Res. Lett. 1 (1994), 399-407. MR 1302384 Zbl 0835.58045

[23] A. Klein, Absolutely continuous spectrum in random Schrödinger operators. In W. Arveson (ed.) et al., Quantization, nonlinear partial differential equations, and operator algebra. 1994 John von Neumann symposium on quantization and nonlinear wave equations, June 7-11, 1994, MIT, Cambridge (MA), U.S.A. Amer. Math. Soc., Providence (RI), 1996, 139-147. MR 1392987 Zbl 0864.60053

[24] A. Klein, Spreading of wave packets in the Anderson model on the Bethe lattice. Commun. Math. Phys. 177 (1996), 755-773. MR 1385084 Zbl 0860.60050

[25] A. Klein, Extended states in the Anderson model on the Bethe lattice. Adv. Math. (1998) 133, 163-184. Zbl 0899.60088

[26] A. Klein, J. Lacroix, and A. Speis, Regularity of the density of states in the Anderson model on a strip for potentials with singular continuous distributions. J. Stat. Phys. 57 (1989), 65-88. MR 1031404 Zbl 0716.60132

[27] A. Klein, J. Lacroix, and A. Speis, Localization for the Anderson model on a strip with singular potentials. J. Funct. Anal. 94 (1990), 135-155. MR 1077548 Zbl 0780.60063 
[28] A. Klein and C. Sadel, Absolutely Continuous Spectrum for Random Schrödinger Operators on the Bethe Strip. To appear in Math. Nachr. Preprint 2011. arXiv:1101.4328v1

[29] A. Klein and A. Speis, Smoothness of the density of states in the Anderson model on a one-dimensional strip. Ann. Phys. 183 (1988), 352-398. MR 0952881 Zbl 0635.60077

[30] F. Klopp, Weak disorder localization and Lifshitz tails. Commun. Math. Phys. (2002) 232, 125-155. MR 1942859 Zbl 1034.82024

[31] H. Kunz and B. Souillard, Sur le spectre des operateurs aux differences finies aleatoires. Commun. Math. Phys. 78 (1980), 201-246. MR 0597748 Zbl 0449.60048

[32] J. Lacroix, Localisation pour l'opérateur de Schrödinger aléatoire dans un ruban. Ann. Inst. H. Poincaré, Phys. Théor. A40 (1984), 97-116. MR 0745684 Zbl 0599.60062

[33] B. Simon, Functional Integration and Quantum Physics. Academic Press, New York etc., 1979. MR 0544188 Zbl 0434.28013

[34] B. Simon and T. Wolff, Singular continuos spectrum under rank one perturbations and localization for random Hamiltonians. Commun. Pure. Appl. Math. (1986) 39, 75-90. MR 0820340 Zbl 0609.47001

[35] W.-M. Wang, Localization and universality of Poisson statistics for the multidimensional Anderson model at weak disorder. Invent. Math. 146 (2001), 365-398. MR 1865399 Zbl 1035.82023

[36] F. Wegner, Disordered systems with $n$ orbitals per site: $n=\infty$ limit. Phys. Rev. B 19 (1979), 783-792.

Received June 7, 2011; revised July 7, 2011

Abel Klein, University of California, Irvine, Department of Mathematics, Irvine, CA 92697-3875, U.S.A.

E-mail: aklein@uci.edu

Christian Sadel, University of California, Irvine, Department of Mathematics, Irvine, CA 92697-3875, U.S.A.

E-mail: csadel@math.uci.edu 